\title{
Clea Laage Die Auseinandersetzung um den Begriff des gesetzlichen Unrechts nach
}

\section{I945}

Es ist bekannt, daß der Versuch, den neu entstehenden demokratischen Rechtsstaat in der Bundesrepublik nach 1945 auf die juristische und politische Aufarbeitung der NS-Vergangenheit zu gründen, gescheitert ist. Nur ein Bruchteil der NS-Tärer wurde bestraft. Ihre Exkulpation implizierte die weingehende Legitumarion des NSStaates und seiner Rechtsordnung. ' Hicrzu gab es eine inzwischen fast vergessene Alternative. Sie bestand in der Kategoric des gesetzlichen Unrechts, die die juristische Aufarbeitung nationalsozialistischen Unrechts 1946/47 prägre, danach aber systematisch verdrängt wurde.

\section{Die Radbruchsche Formel ogesetzliches Unrecht und übergesetzliches Rechter}

\section{Entwicklung einer materiellen Geltungslebre}

Ausgehend von der nach 1945 drängenden Frage, ob z.B. die Beschlagnahme jüdischer Vermögen durch den NS-Staat, Denunziationen und Gerichtsurteile, die sich auf NS-Gesetze stützten, rechtsgültig sind ${ }^{4}$, enrwickelte Radbruch eine materielle, philosophisches Geitungslehre. Danach kommt positivem Recht nur dann Gelcung zu, wenn es dem übergesetzlichen Recht entspricht. Andernfalls liegt "geserzliches Unrecht ohne Geltung vor.

- Wir haben einschen mussen, daß es ein Unrecht in der Form des Geserzes gibr, ein 'gescrzliches Unrecht, und daß nur an dem Maßstab eines übergesetzlichen Rcchis ermessen werden kann, was Recht ist, mag man dieses Recht über allen Gesetzen nun Naturrecht, götliches Recht oder Vernuniluecht nennen, $a^{s}$

1 Frankenberg. Mäller, Jurisusche Vergangenheusbewälugung - Der Volksgenchushof vorm BGH, KJ H. z/19\$3. S. 14s fi.; Friednch, Fresspruch für die Nazi-Jusuz, Hamburg 1983: Bcozler. Justiz und Anstaltsmord nach 1945, KJ H. 2/1988. S. 137 ff.; Kim, Verfassungsumsturz oder Recheskoreinuitäl? Die Stellung der Junsprudenz nach 1945 zum 3. Resch, insbesondere dic Konflikic um die Beamicn. rechte und Ar.131 Grundgescr., Berlin 1972

2 Der Begriff $n$ Radbruchsche Formela zucrst bei Evers, Zum unknuschen Naturrechesbewaßiseun in der Rechesprechung der Gegenviar, JZ 1961. S. 241 (246). Er bezeichnel im engeren Sinne die Unerräglichkerisehese (vgl. unicn), so be Seebode, Das Verbrechen der Rechisbeugung, Berlin 1969, S. 44. Im weiteren Sinne, so ist der Begriff heer verwendet, bezeechnet er die gesamte Radbruchsche Geliungslehre nach 1915; Schumacher, Rezeption und Kritik der Radbruchschen Formel. Diss. Goutungen Ig8s. 5. 24 .

3 Zu beachten ist, daß Radbruchs Lchre vom ubergeseczlichen Recht kein Plädoyer für cane Naturtechtsrenassance im demokratischer Rechisstaat isi. Radbrach betonce: "Ihre Anwendung findet ilure Grenze un den vóllig sıngulären Verhälınıssen der zwoll Nazı-Jalsece, Radbruch, Zur Diskussion über dic Verbrechen gegen dic Menschlichkell, SJZ 1947, Sp. 1 31 ( 136 ).

4 Diesen praxisoricneierzen Ausgangspunke untersireiche Radbruch, Geseizliches Unreche und ubergescexliches Recht, SJZ 1946, S. rosf:; ders, Vorschule der Rechisphilosophie, Heidelberg 1948, S. 1081 . 5 Zum Begriff philosophische Geluungslchre vgl. Radbruch, Varschule .. (Fn. 4), S. 35 .

6 Radbruch, Gesetz. und Racht, Stuuganer Rundschau, H. 1/1947, S. S; cbenso ders., Emeucrung des 
Hiermit gewinnt Radbruch ein Instrument zur Entlegitimierung der NS-Rechtsordnung. Ein Gesctz, das in einem bestimmten Maß' ungercht ist, schafft kein Rechr, sondern ist "gesecicliches Unrechta ohnc Geltung. Durch diese Entlegutmierungsfunktion der Kategorie des gesetzlichen Unrechts wird die Ahndung von NSVerbrechen (Bestrafungsfunktion) möglich ${ }^{8}$, denn Nichtgeltung geseczlichen Unrechts impliziert, daß der Mensch im Falle eines Konflikts zwischen positivem Gesetz und übergesetzlichem Recht verpflichtet ist, sich an letzterem zu orientieren und dem gesetzlichen Unrecht den Gehorsam zu verweigern.

. Veın Geserze den Willen zur Gerechugkeı bewußt verlcugnen, ... dann schuldet das Volk ihnen keinen Gehorsam, dann müssen auch Jurssten den Mut finden, ihnen den Rechuscharakter abzusprechen. $x^{y}$

Befolgr jemand dennoch das positive Geserz, bleibt seın Handeln rechrswidrig, da es dieses nicht rechtfertigen kann.

Mit beiden Funktionen erwcist sich die Kategorie des gesctzlichen Unrechts als Alternative zu der 1947/48 einserzenden Restauration, die gerade auf der Legitimation des NS-Staates und seiner Rechcsordnung beruhte und deren Kernstück die Nicht-Ahndung von NS-Verbrechen war. ${ }^{10}$ Die Kategorie des gesctzlichen Unreches stehr im Spannungsfeld Neubeginn versus (Rechts-)Kontinuitär auf dor Seite des politischen und rechtlichen Neuanfangs.

2. Wann ust ein NS-Gesetz "gesetzliches Unrechta?

Zur Abgrenzung geseszlichen Unrechts von trotz unrichtigem Inhait dennoch geltenden Gesetzen entwickelte Radbruch die sogenanntc Unerträglichkeitsthese."

Rechts, in: Gedächtnusschrift fur Guseav Radbruch, hg. v. $\Lambda$. Kaulmann, Göungen r 968, S. 26; ders., Dic Erncuerung des Reches, in: Maihofer (Hg.), Naturrecht oder Rechesposutvismus?, Bad Homburg $1962, S, 2$.

Das Verhälenss von übergesetziichem Reche und Naturrecht ist bet Radbruch nicht klar. Einersetts serzt or ubargesetaliches Recht mit Vernunftrecht, Naturrecht und goulichem Recht gleich (Radbruch, Grsetx und Reclu, ebd., S. ५). Anderersets waml or vor der Gleichsetzung von Naturrecht und übergescizlichem Reche. da leizterem >nicht die übemationale, überzestliche Universalität des Naturreches der Vergangenhoit a zukomme, sondem es ein "Naturrechi mir wachscindern inhali, rvandelbar mach Zcil und Volka ses (Radbruch, Neuc Probleme der Recheswissenschale, in: ders., Eine FeucrbachGedenkrede sorvie drei Aufsaize aus dem wissenschafelichen Nachlaß, hg. v. E. Schmide, in: Recht and Suat in Geschiche und Gegenware, H. 172, Tubingen 1953, S. 32 f).

7 Vgl. dazu unten dic Unerräglichketsihese.

8 Perels, Die Restaumation der Recheslehre nach 1945, K] H. ${ }_{4} / 1984$, S. 359 (363); a. M. Engisch, Gustav Radbruch als Rechtsphilosoph, ARSP 1949/50, S. 305 (315): wan würdc Radbruch sehr mißdeucen. wollte man annehmen, er habe hicr cine Theorie als Walfe geschnouder gegen die verabscheute Vergangenhert. ... Um Abrechnung mil der Vergangenhen war es Radbruch nuche zu tun.*

9 Radbruch, Füni Minuten Rechtsphilosophie (1945), in: ders, Rechtsphilosophic, 8. Auf., hg. v. Woir. Schncider, Stuitgart 1973, S. 32 S.

10 Vgl. xu dicscm Rescaurationsbegrifl Percls, (Fn. 8), S.j601. Wertere vescntliche Detcrminanten des Resiaurauonsproecseses sind:

erstens die Übernahme der NS-Trägerschichıcn in die Exekutıve und Jusuz der Bundesrepublik: Vgl. daxu Wenzlau, Der Wiederaufbau der Justiz in Nordwestdeurschland r945-1949, Kómgstem 1979 Feest. Dic Bundesrichter. Herkunft, Karmere und Auswahl der jurisuschen Elice, in: Zapf ( $\mathrm{Hg}$.) Besträge und Analysen zur deutschen Oberschehe, 2. Aufl., Munchen 1965, S. 95 If.; Fangmann, Dic Ristauration der herrschenden Statssechtswissenschaft rach 194s, in Reifner ( $\mathrm{H}_{\mathrm{g}}$ ), Das Reche des Unruchesstazics, Frankfurt a. M. 1981 , S. 211 If.;

x.wertens dic Ablosung der Kondominiumscheone Kelscs (Vgl. dazu: ders., The International Legal Status of Gemmany 10 be Established Immediately upon Tcrmination of the W/ar, in: A mecican Journal of Law, Bd. $3^{8}$ (1944), S.689ff.; ders., The Legal Status of Germany 10 the Declaration of Berlin, in: Amencan Journal of Law, Bd. 39 (1945). S. s18ff.) durch die Idertulätsethcone (vgl. Siödecr. Deueschlands Rechtslagc, Hamburg 1948, S. 361 .;.; Greve, Ein Besatzungsseatur für Deutschland, Stutegart 1948, \$. 47 lf. bes. S. 77 If: Kauimann. Deueschlands Recheslagi unter der Besaczung. Sevigan 1948):

destens das Scheitcm der Neuordnung der Winschafesstrukeur, vgl. dazu Winter, Sozialisierung in Hessen 1946-1955, KJ H. 2/1974, S. r 97 If, Zur Diskussion uber dic Kontunurä̌ Kocka, Rescuuraion oder Neubeginr)? Deurschland 1945-1949, L'76, H.11/979, S. 112 ff. m. w, N.

II Mit Unererügliclikeisshesc bezeichne' Schumacher, (Fn. 2), S. 25 folgende Passage in Radbruchs 
Da nach Ansicht Radbruchs jedes Gescrz - also auch das narionalsozialiszische -

Rechtssicherheir garantiert'2, bestcht cine Vermutung für die Geltung auch des ungerechien und unzweckmäßigen Geseczes.

"Der Konflikt zwischen der Gerechrigkes und der Rechessicherhcie dürfte dahın zu lösen sein. daß das positive, durch Satzung und Macht gesicherte Recht auch dann den Vorrang hat, wenn es inhaltlich ungerechr und unzweckmäßig ist. $\alpha^{\text {"s }}$

Gesetzliches Unrechi sei deswcgen inncrhalb jedes Rechtssystems die Ausnahme:

- Wern sich also in der Regel der Fälle die Geleung posiuven Rechts durch die Reclussicherhcit rechtfertigen läßt, so blcibe in gewissen Ausnahmefällen horrend ungexecher Gesetze die Möglichkeit, solchen Gesetzen ihrer Ungerechtigkeit wegen die Geltung abzusprechen. « $^{\text {'s }}$

Geserzliches Unrecht liegt erst vor, wenn

- der Widerspruch des posutuven Geseczes zur Gerechtigkeıt ein so unererägliches Maß erreichı, daß das Gesetz als sunrichuges Rechu' der Gerechnigkcit zu weichen hat.a"s

Ergänzt wird die Unerträglichkeitsthese durch einc zweice von Radbruch benutzte Formel zur Abgrenzung von geltendem und nicht-geltendem Recht, dic sogenannte Verleugnungsthese. ${ }^{16}$ Sie laucet:

"Wo dic Gercehrigkeit nicht einmal ensurcbe wird, wo die Gleichheit, dic den Kern der Gerechrigkeit ausmacht, bei der Setzung positiven Reches berwußr verleugnet wurde, da ist das Gesetz nicht etwa nur unrichtiges Recht, vielmehr entbehrt es überhaupt der Rechisnacur. Denn man kann Recht, auch positives Recht, gar nicht anders definicren, denn als cine Ordnung und Satzung, dic ihrem Sinn nach bestimme ist, der Gerechtigkeit zu dienen. $x^{\prime \prime}$

Da die Verleugnungsthese das einzelne NS-Gesetz in Bezichung setzc zur aligemeinen rechtsverieugnenden Intencion des nationalsozialistischen Gesetzgebers, ${ }^{18}$ ist "gesetzliches Unreche nicht mehr der Ausnahmefall, sondern

*[a]n diescm Maßstab gemcsscn sind ganze Partien nationalsozialisuschen Rechts nicmals zur Würde geitenden Rechts gelangt. $\alpha^{19}$

Die Unerträglichkeits- und die Verleugnungsthese, der Gerechtigkeitsbegriff und der Gleichheitssacz sind bei Radbruch bezogen aul die "Humanität als Reclitsbegriff $\pi$, d. h. "Menschenfreundlichkeit gegen unmenschliche Grausamkeit, ... Menschenwürde gegen unmenschliche Erniedrigung, ... Menschenbildung gegen unmenschliche Kulturvernichtung, ${ }^{20}$ und auf die Menschenrechre, in denen die Humanität zum Rechtsbcgriff wurde." Daraus ergeben sich zwei weitere unum-

Aufsariz, Geserzliches Unreche..., (Fn. 4), S. 107 1.Sp.: "Der Konflikt zwischen der Gerechugkut und der Rechussicherhes durlte dahın zu lösen seın, daß das passeve, durch Sazung und Mache gesichere Recht auch dann den Vorrang hat, wenn es inhalelich ungerecht und unzweckmäbig ist, es set denn, daB der Widerspruch des positiven Gesetzes zur Getechtigkeis ein so uncriagliches Maß erreicht, daß das Gesetz als unrıchuges Recht، der Gerechtıgkeıt 7.u weıchen hit. Es ıst unmöglich, eine schärferc Lunıe zu zuehen zwischen den Fällen des gescuzlichen Unrechts und den trotz unnchugen Inhalis dennoch getenden Gesctzen. «

12 Radbruch, Gesezzliches Unrecht..., (Fn, 4), S. 107 1. Sp.: "[F]inen Wen fühn schon jedes Gesetx ohne Rucksiche auf seinen Inhalt mit sreli. Es ise immer noch besser als kein Geseiz. wcil es zumindese Rechessicherhe'l scbaifi."

1) Ebd.

14 Radbruch, Vorschule..., (Fn. 4). S. 35; cbenso ders., Neuc Probleme... (T.n. 6). S. 33: Dic Annahme gesetzlichen Unrechis ist beschrankt auf wFälle esnes flagranten, liur ntemand ernsilich bestreitbarcon, schlechihin verbrecheriscisen MjißBbrauchs in Gesetzesform.*

is Radbruch, Gesetzliches Unreclic.... (Fn. 4). 5. 107 I. Sp.

16 Vgl. Schumacher, (Fn. 2), S. 26

17 Radbruch, Gesetzliches Unrecht..... (Fn. 4), S. 107 r. Sp.

18 . Dit hervorstechendse Eigensehafe in Hillers Persönlichker, die von ihm aus zum Wesensxuge des ganxen nationalsozialisusehen, Rechise wurde, war sein völliger Mangel an Wahrheitssinn und Rechr5$\sin n \alpha$. Ebd, hervorh. C.L.

19 Fbd ; der Widerspruch zwischen Ungütugkent gavzer Partien des NS-Rechts und dem Ausnahmecharakier gesetzlichen Unrechts bleibe bei Radbruch unverminelt nebeneinander stehen.

zo Radbruch, Vorschule..., (Fn. 4), S. $94 \mathrm{f}$

21 Ebd., S.91. 
stößliche Kriterien für das Vorliegen geseczlichen Unrechts. Gesetzliches Unrecht liegt somit vor:

- bei ${ }_{n}$ Gesetzen, die Menschen als Untermenschen bchandeln und ihnen die Menschenrechre versagten «,

- bei Gesetzen, die Menschen zum Objekt staaclichen Handelns emiedrigen, also bci all »jenen Strafdrohungen, die ohne Rücksicht auf die unterschiedliche Schwere der Verbrechen, nur geleitet von momentanen Abschreckungsbedürfnissen, Straftaten verschiedener Schwere mit der gleichen Strafe, häufig mit der Todesstrafe, bedrohten. $\otimes^{23}$

Die Radbruchschic Formel kann durch diese Betrachtung des aus der NS-Rechtsordnung isolierten einzelnen Gesetzes nur eine punktuelle Entlegitimierung der NSRechtsordnung erreichen. ${ }^{4}$ Deswcgen muß sie crweicert werden. Die Frage nach dem geserxlichen Unrecht muß bezogen wcrden auf die Frage nach der demokracischen Legitimität bzw. Illegirimität des nationalsozialistischen Gesetzgebers und auf dic Frage nach dem Charakter der ganzen NS-Rechtsordnung und der Rechrspraxis." Die Radbruchsche Formel bedarf der Einbindung in die materiellen Analysen des Unrechis-Sraaces ${ }^{26}$ von Fraenkel, Kirchheimer und Neumann ${ }^{2 y}$ unter dem Gesichtspunkt, ob den NS-Gesetzen - wie Radbruch unterstellt ${ }^{18}$ - Rechtssicherheic als Wert innewohnt, der eine Vermurung für deren Gülcigkeit rechterrigt. Nach Fraenkel ist die "Rechtsordnung des Doppelstaates « durch das Nebeneinander von Normen- und Maßnahmenstaat gekennzcichner. ${ }^{29}$ Der Maßnahmenstaat, das Herrschaftssystem der unbeschränkten Willkür und Gewalr, ${ }^{30}$ kann durch die Definition des seiner Herrschaft unterstehenden politischen Bereichs den normenstaaclichen. Rechosbereich auf Null reduzieren, mic der Folge, daß iendenziell die gesamte Rechtsordnung weder Rechtssicherheit noch Rechtsschurz garantier. ${ }^{31}$

Wahrend Fraenkcl die Ncgacion eines Rechtssicherheit gewährenden Rechrs nur als Möglichkeit bezeichnet, die 1938 erst zum Teil Wirklichkeit geworden sei, ${ }^{32}$ kommen Kirchheimer und Neumann zu dem Ergebnis, daß es im NS-Staac kein Rechtssicherheit garantierendes Recht gab. Der nationalsozialistische Staat degradierte das Recht zum rejnen Funkcionsmodus der Macht, von Recht, das wnicht nur voluntas, sondern auch ratio a ist, könne keine Rede sein. "Bei Neumann heißt es:

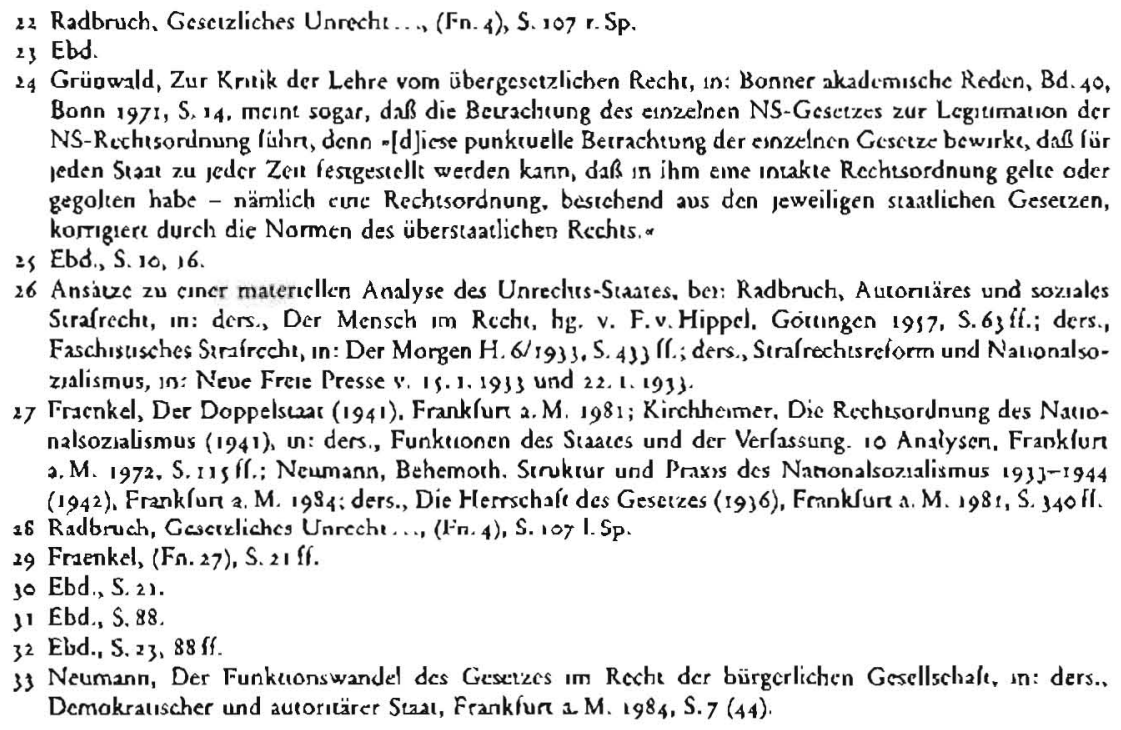


„Es gibr heute in Deutschland kern Recht mehr. Was Recht genannt wird, ist ausschließlich eine Technik, um den politischen Willen des Führers in Ver\{assungstwirklichkeit zu Iransformieren. «st

Kirchheimer Kaßr den Gedanken noch genaucr:

-[D]as okonomisch atomisienc Individuum wird zum bloßen Objekı der Herrschaft von monopolistischen Gruppen und Staatsmaschinerie. Gleichzeitig verlien dic Legalizat ithre Funktion als Walfe zum Schutz des individuums. Sie wird völlig bedcutungslos und löst sich in zechnısche Rationalität auf. Diese ist nunmehe das Strukıurprinzip der Rechtsinstieutionen, des Gesetzgebungsapparates und des Anwendungsinstruments, der Richterschaft, ass

Vor diesem Hintergrund kann davon, daß die Rechtssicherheit in der Regel die Geltung nationalsozialisuscher Geserze rechtfertigt, nicht gesprochen werden. Zumindest ist das nunerträgliche Maß schnell erreicht, wenn ein Gcsetz weder Rechtssicherheit schafft noch Gerechtigkeic intendiert. Eine Vermutung für die Geltung positiven Rechrs kann es nur im rechisstaatlichen Staatswesen, nicht für den Rechrssicherheit und Gerechtigkeit verleugnenden NS-Unrechts-Staat geben.

\section{Alliierte Gesetzgebung im Sinne der Radbruchschen Formel}

\section{Eninazifizterung der NS-Rechcsordnung}

Der Umgang der Alliicrten mit dem NS-Rechtssystem ist gekennzeichnet durch die Kombination von drei Strategien, die die Kontinuität zwischen NS-Rechesorónung und neuem Recht unterbinden sollen:

a) Außerkraftsetzung der Grundgesetze des NS-Staates,

b) Fesilegung von Anwendungs- und Auslcgungsrcgeln für das inkraftbleibende NS-Rech ${ }^{36}$ und

c) Handhabung des Rechts durch eine sentnazifizierter demokratische Justiz.

Das Militärregierungsgesetz $\mathrm{Nr}$. 1 (MilReg 1$)^{37}$ und das Kontrollratsgesetz Nr. 1 (KRG 1) vom 20.9. $1945^{38}$ setz(e) die 25 wichtigsten "Gesetze politischer Natur oder Ausnahmegesetze, aul welchen das Nazi-Regime beruhte", außer Kraft. Aufgehoben wurden das Ermächrigungsgesetz, viclc judendiskriminierende Gcsetzc $^{39}$ und Geserze, die dem Schurz des NS-Sraares und der NSDAP dienten. Dieser offene Kacalog ${ }^{40}$ wurde durch das KRG I vom 30. 1. $1946^{41}$ ergänzt, das u.a. den $\$ 2$ StGB, der die strafrechtliche Analogie und die Bestrafung nach mgesundem Volkscmpfinden «zuließ, die Hoch- und Landesverratsbescimmungen ( $\$ \$ 80-94$ $\mathrm{StGB}$ ) und das diskriminierende Sonderstrafrecht (z. B. die Kriegssonderstralrechtsverordnung ${ }^{22}$, die Volksschädlingsverordnung ${ }^{-13}$, die sogenannte Polen- und Judenstrafrech(sverordnungs') aufhob.

Die Flandhabung des weitcrhin geltenden nationalsozialiscischen Rechts wurde

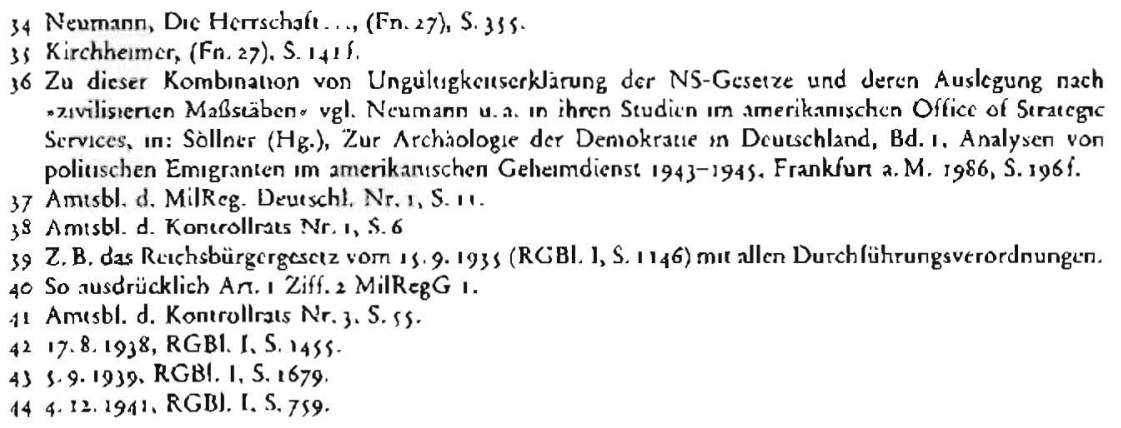


durch die Formulierung allgemeiner Anwendungs- und Auslegungsvorschriften im MilRegG I, im KRG $\mathrm{r}$ und in den Allgemeinen Anweisungen der Militärregierung an Richter Nr. I's geregcle:

a) Die Auslegung und Anwendung des Rechts nach nationalsozialistischen Lchren oder unter Bezugnahme auf Gerichtsentscheidungen oder Schriften, die nationalsozialistische Gedanken vertreten, wurde verboten. ${ }^{46}$

b) Das weiterbestehende NS-Recht war ventsprechend dem klaren Sinn des Wortlauts auszulegen und anzuwenden 4.47

c) Die Bestrafung unter Anwendung von Analogie oder nach angeblich ngesundem Volksempfinden $\alpha$ wurde untersagt. . $^{\text {s }}$

Abgesichert wurden diese Auslegungs- und Anwendungsvorschriften dadurch, daß die Handhabung des nicht außer Kraft gesetzten NS-Rechts in die Hände einer entnazifizierten, demokratischen Jussiz gelegt werden sollte. Die ordentlichen und die Verwaltungsgerichte wurden bis auf weiteres ${ }^{49}$ geschlossen..$^{\circ}$ Der Volksgerichishof, die Partei- und Sondergerichee wurden abgeschaff." Das KRG 4 s: verfügte die Amtsenthebung der Richtcr und Staarsanwälte, die sich als Mitglieder der NSDAP akciv für deren "Tätigkcit cingeseız hatten..$^{53}$

In der Kontrollratsproklamation Nr. 3 wurden dic Grundsärze der neuen Justiz festgelegt. Sie mußte sich orientieren an Demokratie, Gerechtigkeit, Gieichheit vor dem Gesetz und den Grundsä̈zen eines fairen Verfahrens.'s

\section{Das Kontrollratsgesetz Nr. $10^{\text {ss }}$}

Parallel zur Entnazifizierung des NS-Rechessystems und der NS-Justiz vollzog die alliierte Geserzgebung den rechelichen und politischen Bruch mit dem NS-Staat und sciner Rechrsprechung vor allem durch das Kontrollrarsgeseiz Nr. 10 (KRG 10). Das Kontrollrarsgesetz gab der deutschen Rechrspilege eine Chance, cincr Zcit revolurionären Unrechts... durch revolutionäres Rechr Herr zu werden. ${ }^{86}$ Ar. II Ziff. ıa-d KRG ro erklär vier Tatbestände für strafbar: Verbrechen gegen den Frieden, Kriegsverbrechen, Verbrechen gegen die Menschiichkeit und die Zugehörigkeit zu verbrecherischen narionalsozjalistischen Organisationen. Das Verbrechen gegen die Menschlichkeirs7 ist als Positivierung der Radbruchschen Formel zu

4) SchlHA 1946, S. 4 .

46 An. III MilRegG I, Amisbl. d. MilReg. Deulschl. Nr. 1, S. 11

47 Ebd.

$4^{8}$ An. IV Ziff. 7 MilRegG 1, ebd. Ob damut alle Strafvorschriften, die die Fomulierung agesundes Volksempfindenx enthelten, unanwendbar wurden, ob stch also sus dieser Auslegungsvorschrift eine Nicht-Anwendungavorschrift abletten läßt, vgl. Fn. 73 und III.t.bb. (2)

49 Ar.IXI, IV MilRegG 2, Amesbl. d. MilReg. Deutschl. Nr. 1, S. 13.

so Arr. III der Proklamation Nr. I, Amesbl. d. Milkeg. Deuuschl. Nr. I, S. 1; Art. I MilRcgG 2. Amesbi. d. MilReg. Ueutsclil. Nr i, S, is.

Si Ar. ll MilRegG 2, ebd.

s2 Amusbl. d. Koncrolleats Nr. 2, 5. 26.

s3 Zu diesem Ansau esnes qualitauven Entnazifizierungskonzepts auch Direkuve Nr. 24, Zill. 1 u. 2 v. 12.1. 1946, Amesbl. d. Koncrollints Nr. 5, S. 26.

s4 Amtsbl. d. Konrolirats Nr. 1, S.22. Einen cntsprechendon Eid hateen Richter, Staatsınwälte, Notare and Rechisanwiblic zu lessten. Art. $\vee$ Ziff. 8 MilRegC 2, Amesbl. d. MilReg. Deuischl. Nr.1, 5. 13.

ss Amtsbl. d. Kontrolleass Nr. 3, S. so.

s6 Baucr. Das "gesetzliche Unrechu des Nationnlsozialismus und die Survfrechispflege, in: Gedachinisschpif, fur Gustav Radbruch, hg. v. A. Kaufmann, Götungen 1968, S. 302 (307); ahnlich Kim. (Fn. 1). S. 74 .

57 Das Verbrechen gegen die Menschlichkeı kann in dreı "den... Tubestand ,edoch nelur erschöplende» (Art.II Ziff.se KRG 10) Trebestandsgruppen gegliedert werden:

s) such im SiGB gebräuchliche Delikusbezerchnungen, wie Mord, Freshertsberaubung und Vergewalı1.

gung
b) Massenverbrechen: Ausroutung, Versklavung, Zwangsverschleppung oder andere an der Zivilbevolkerung begangene unmenschliche Handlungen 
verstehen. ${ }^{8}$ Denn Anknüpfungspunkt der Strafbarkeit ist nicht das staatliche Geserz, sondern das überpositive Rechr. Geschürztes Rechusguc sind die Menschenrechte, die Menschheit als Trägerin dieser Rechte." Dieses Schutzgut wird vom Obersten Gericlushof für die britische Zone $(\mathrm{OHG})^{60}$ in prägnanter Weise in die Formulierung des obiektiven Tatbestands des KRG 10 aufgenommen. Er fordert, daß Menschen oder Menschenrechte angegriffen oder geschädige werden und daß dicse Schädigung in einer Weise geschieht, die die "für Nichts-Achtung des ideellen Menschenwerts mit Wirkung für die Menschheita ausdrückt. ${ }^{61}$ Diese geforderte überindividuelle Wirkung ist gegeben, wenn die Tat

nın ihrem Gesamehergang, abgesehen von der Rechisgüterverleızung in bezug auf das Opfer, zugleich auch allgemcin als grundlegend anerkannte Kulturgütcr verletzt, wic elwa die im europäschen Rechiskress unabdingbarc Glcichheit aller Menschen ohne Untersehico der Rasse und des Bekenntnisses vor dem Gesctz.... ferner, wenn die Tat die innerhalb rechrsstaztlicher Grenzen garantıerte Freiheit der politischen Meinungsbildung und Meınungsäußcrung verlcizl. Dic Menschhcit ist ferner berühr, wenn die Tat ... darübcr hinaus in einem human denkenden, rechisstantich organisienen Volke als Antworr au das konkrete Verhakten des Opfers objektiv in dieser Art und Schwere nicht denkbas wäre. ${ }^{\sigma_{2}}$

Neben dem gemeinsamen Schurzgur ergibt sich der Zusammenhang zwischen KRG ro und dem Begriff des gesetzlichen Unrechts daraus, daß beide davon ausgehen, daß die Verletzung der Menschenrechte auch dann Unreche bleibt, wenn sie geserzlich gebocen ist.

„Der Versioß gegen dicsc Grundsätze der Menschljelikeil bleibr surafbarcs Unrecht, auch wenn er von emem Stant geduldet, geförder oder veranlaßt wird. ... Das Kontrollratsgesetz Nr. 10 gcht davon aus, daß dieser, gewisse Grundsäızc der Menschliclikeit umfassende Kernbereich des Rechis durch innerstazlliche Gesctze und ilure Handhabung nicht abgewandelt werden kann. $x^{63}$

Indem das KRG 10 die Einwendungen der Angeklagten - sic seien durch das NaziRegime begnadigt oder amnestiert worden oder sic hätten auf Befehl der Regierung oder eines Vorgesctzten gehandelt - für irrelevant erklär $\mathrm{c}^{64}$ und indem es bestimmt, daß Verbrechen gegen die Menschlichkeir unabhängig davon vorliegen, ob die Taten "das nationale Recht des Landes, in welchem die Handlung begangen worden ist, verletzen ", ${ }^{6}$ schneider es jede Berufung auf die NS-Rechtsordnung, auf ein dem übergeserzlichen Recht entgegenstehendes nationales (Un-)Rechr ab.

"Es gehörı nicht zu den Voraussetzungen des Verbrechens gegen die Menschlichkeit, daß dic Handlung auch von der innerstastichen Rechtsordnung eindeutig als strafbares Unrceht

c) Verfalgung aus poliuschen, mssischen oder religiösen Gründen.

Zu dicser Tatbesiandsgruppierung vgl. Gude. Die Anivendung des Kontrolleatsgeserzes Nr. 10 durch dcutsche Genchtc. DRZ 1947. 5.111 (11).

s8 nAuch dieses übergescizliche Rechı kann Gesetzesform annelimen... solcher Ar sst das KRG 10.* Radbruch, Gesev\% und Recht, (Fn, 6), S. s.

59 Deswegen übersetzt Radbnuch "Cnmes agannst Humanity a mit Verbrechen gegen die Menschhert. Radbruch, Zur Diskussion uber die Verbrechen gegen die Menschlichkerc, SJZ 1947. Sp.131 (133).

6o Der OGH (Tätigkent von Fruhpahe 1948 bis 1950) wat zunächst nur mit Gcgnem des Nutionalsozıalismus besetze: E. Wolff (1938 aufgrund der nationalsozialisuschen Rassegeselkgebung als Rechesanwalt in Berlin gelöscht, nach London emigner), A. Wimerer ( $193^{8}$ aus poliuschen Grunden in der Ruhescand versetzi), K. Staff (nacla der.Macheergreifung. durch den Nacionalsoxılismus aus dem Jusuzdicnse entlassen). Er vurde dann erwercert durch Richicr, die teilwerse auch zowischen 1933 und 1945 als Rechisanwäle oder in Ministerien taug gewesen waren, vgl. ZJ日L. 1/1948, S. 7.

61 OGHSI I, 11 (Leitsaiz) v. 20.5.1948; 1. 19 (20f.) v. 22.6.1948; 1, 36 (38) v. 13.7.1948.

62 OGH5t 1, 43 (Lentsätzc ! und 3) v. 27.7.1948; 1,11 (14 f.) v. 20. 5. 19,18.

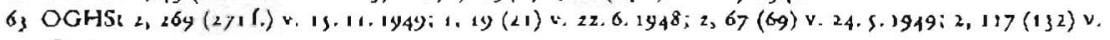
18. 10.1949.

6 A Ar. II Zill. b. Ziff.s KRG 10

6s An. Il Ziff. ic KRG 10. Es 1st nıche ersechrlich, warum dicse Klausel nur beım Verbrechen gegen die Menschlichkeit stcht. Tatsïchlich findet der in ihr enthalienen Gedanke nach dem Willen des Geseczes zuf sämtliche in KRG to enthaltener Tabesiande Anwendung, vgl. Kmus, Kommenuar zum Kontrollmisgestes Nir. 10, Hamburg 1948, 5.80. 
gewürdige wird, es ist vielmehr gerade der Sinn und Zweck des KRG 10, daß Verletzungen jener Grundsäıze der Menschlichkeit als Verbrechen im Sinne des Arr. II ic KRG 10 auch dann bestraft werden, wenn die Handhabung der sratichen Rechusordnung sie straflos ließ oder sie gar begünstigne. Das Kontrollratsgesęz Nr. 10 forderı d́abei von jedem, daß er sich für die Grundsätze der Menschlichkeir entschied, wenn ihm cin stäatliches Geselz oder scine Handhabung die Möglichkett bot, sie zu verletzen. ${ }^{66}$

\section{Dic Ausetnandersetzung um den Begriff des gesetzlichen Unrechts}

\section{Was sind typisch natonalsozialistische Gesetze?}

Ob cin Gesetz stypisch nacionalsozialistisch" ist, "typischen Nazigeist enthält oder nausgesprochen nationalsozialistische Ziele verfolgıe, so lautete die Frage, die Rechtsprechung und Rechrslehre nach 1945 stellten, um zu entscheiden, ob ein Gesetz als geserzliches Unrechi unanwendbar geworden war. ${ }^{67}$ Diese Fragestellung schränkte Radbruchs Überlegungen zur Geltung nationalsozialiscischer Gesezze an einem entscheidenden Punkt ein. Während Radbruch meinte, daß geseczlichem Unrechr von Anfang an (ex tunc) jede verbindliche Kraft lehite, so daß aul solche Gesctze gestützte Handlungen schon in der NS-Zeit rechtswidrig waren, ziclec die Frage nach dem typisch nationalsozialistischen Gcsetz nur auf die Zukunft. Gefragt wurde allein, ob ein Gesetz nnicht melur anwendbar ${ }^{68}$ sei. Soll die Geltung dem Gesetz nicht auch rücksvirkend versagt werden, entfällt die Bestrafungsfunktion der Radbruchschen Formcl, demn dann waren die diesen Gesetzen entsprechenden Handlungen damals rechrmäßig.

Parallel dazu einigte man sich in den Westzonen schon Anfang r 947 darauf, daßentgegen der Intention der alliicrten Geserzgebung ${ }^{69}$ - die Aufhebung von NSGesetzen durch die Alliierten nur für die Zukunít (ex nunc) svirke. ${ }^{70}$ Begründet wurde dies mit der andernfalls entstehenden Rechtsunsicherheit, sprich: mit dem Wunsch, eine Unterbrechung der (Rechts-)Koncinuität selbst bei geset\%lichem Unrecht zu verhindern.

- Es würde zu einer ungeheuren Verwirrung und Rechısunsıcherheit führen, wenn man der heurigen Anschauung entgegenstehende Gesetze des narionalsozialistischen States als meemals vorhanden gewesen betrachten und alle dadurch entstehenden Rechesverhältnisse so beurceilen wollte, als ob es diese Gesetze nıemals gegeben häue. Gerade auf dem Gebicte des Wirrschaftslebens, das durch die einschneıdenden Maßnalumen der Arssienung ... betroffen worden ist, wären die Folgen höchst unheilvoll. ${ }^{72}$

a) Das Prüfungsrecht des Rucbiers

Da die Alliierten die NS-Rechtsordnung nur ciner sehr lückenhaften Revision unterzogen hatten, ${ }^{72}$ stellte sich die Frage, ob die Richter weitere Gesetze außer Kraft setzen durften. In Übereinstimmung mit dem $1946 / 47$ noch herrschenden

66 OGHSI 2, $269(272)$ v. 15.11.1949.

67 OLG Braunschwe1g 7.6.1946, SJZ 1946. S. 119: OLG Frciburg 12.9.1946, DRZ 1947, S. 65 ; OLG Kiel 22.1, 1947, DRZ 1947, S. 198: OLC Ncustadt 4. 8. 1949, MDR 1949, S. 706; OLG Kassel 29.9.1948. NJW 1949, S. 38 s (386); v. Weber, Reche und Pflicht des Richters zur Prüfung der Gultigkert des Siralgesetzes, in: Tagung deutscher Juristen, Bad Godcsberg, Sonderverölf. des ZJBl. für die britische Zone, Hamburg 1947. S.163 (167)

68 OLG Braunschweig 7.6.1946. SJZ 1946, S. 119; OLG Frciburg 12.9. 1946, DRZ 1947, 5.65.

$69 \mathrm{OGHZ}_{4}, 121,(124 \mathrm{l}$ ) v. 5.7. 1950.

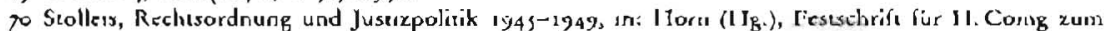
70. Giburstag. Bd. 1., Munchen 1981, S. 383 (401); OLG Kiel (Garbe-Fall) 26. 3, 1947, SJZ 1947, Sp. 323 (327): LG Hagen 1. 3.1947, MDR 1947. S. 29 (30); OLG Saarbrücken 21. 5. 1947, DRZ 1947. S. 341 (343), mIl Anm. Coing. DRZ 1947. S. $342 ;$ 2. M. OGHZ 4 , 121 (124) v. 1. 7. 1950.

7: LG Hagen 1.3.1947, MDR 1947, S. 29 (30); ebenso OLG Sarbrucken 21. 5. 1947, DRZ 1047, S. 341.

72 Werigler, Die Nichunwendung natoonalsozialisuschen Reches in) Lichie der Rechisvergleichung und der allgemeinen Recheslehre, JR 1949, S.77: Wenylau, (Fn. 10) S. 14s; Srollas, (Fn. 70), S. 388. 
Standpunkt einer angestrebten Unterbrechung der Kontinuitäc zwischen NSRechtsordnung und neuem demokratischen Recht befürworete die Rechisprechung - gestützt auf Art. III Ziff. > MilRegG $1^{73}$ - zu diesem Zeitpunkt ein Prüfungsrecht des Richters. ${ }^{74}$

Die gegenteilige Auffassung - der Richter könne kcin NS-Recht außer Kraft scczen ${ }^{7 r}$ - konnte erst 1948/49 vordringen im Zusammenhang mit der sich durchserzenden Auffassung, daß der vor 1945 bestehende Scaat und damit auch seine Rechrsordnung weiter existierte. ${ }^{\gamma 6}$

Problematisch wurde das Prüfungsrecht erst dadurch, daß die van den Alliierten für den adäquaten Umgang mit dem NS-Recht gemachte Voraussetzung - die Handhabung dieses Rechss abliegt einer neuen demokratischen Justiz - durch die Übernahme von NS-Juristen in den Justizapparat der Bundesrepublik"7 entfiel. Von dieser Justiz war die Aufhebung weiterer NS-Gesecze als ntypisch nationalsozialistisch" nicht zu erwarten, da dies immer das Verdikt implizierte, damals selbst Unrechts-Geserze angewandt zu haben.

b) Die Ausübung des Prüfungsrechis

aa) Bekenntnisse zur Radbruchschen Formel

Ab 1946 bekannten sich Rechtslehre und Rechtsprechung mehrheitlich - of unabhängig von der konkreten Prüfung eines NS-Gesetzes - zur Radbruchschen Lehre vom übergesetzlichen Recht und geserzlichen Unrecht. ${ }^{8}$ Die Radbruchsche Verleugnungsthese fand - fast wörtlich - Eingang in die sogenannte Kernbereichstheorie des $B G H$ :

"[I]m Bewußsein aller zivilisienen Völker [besteht] ein gewisser Kernbereich des Rechıs, der nach allgemeiner Rechusüberzeugung von keinem Gesetz und keiner sonsrigen obrigkeitlichen Maßnahme verletzt werden darf. ... Der Senat hat deshalb in der angeführten Entscheidung [BGHSt 2, 234] ausgeführ, daß Anordnungen, die die Gereclutigkeit niche einmal anstreben, die den Gedanken der Gleichheit bewuße verleugnen und dic alden Kulturvölkern gemeinsamen Rechtsüberzeugungen, die sich auf den Wert und dic Würdc der menschlichen Persönlichkeiı beziehen, deullich mißachten, kein Reche schaffen und daß ein solchen Anordnungen entsprecliendes Verialten Unrechı bleibi. *\$

7j Obwohl Ân. llI Ziff. 7 MilRegG I, der die Anwendung und Auslegung des Rechts nach natıonalsozıalisuschen Lehren verbietet, eine Auslegungsregel ist, entspricht die Ableitung anes richterlichen Prüfungsrechts vermuelich dem Willen der Allicenen. Denn es wäre widersinng anzunehmen, daß die Alliieren dem Richter zwar verbouen häucn. Geseıze im natıonal sozıalisuschen Simn auszulegen, daß es ilım aber anderecseits niche crlaubi sei zu prüfen, ob niche ein Geseiz, bereits nach dem klaren Sinn des Wortaus nationalsozialisuschen Gesst wicdergibe und deshalb uneragbar sei. Zu diescr Argumcniawon: Wengler, (Fn. 72). S. 77 1.Sp.; BVeriGE 6. 389 (417).

74 KG Berlin 29.12.1946, JR 1947. S. 27; OLG Frciburg 12.6.1946, DRZ 1947, S.69; LG Celle 20. 12.1946, Hann. Rpfl. 1947, S. 14; OLG Kiel 22, I. 1947, DR2 1947. S. 198: OLG Tübingen 24.2.1947, DRZ 1947, S.164; gegen cen Prüfungsrechl zu dicsem Zelepunke nur OLG Hamburg 26. 3. 1947, MDR 1947, S. 137.

75 Insbesondere ließe sich em Prifungsrecht nicht aus Ar. III Ziff. 7 MilRegG I herleıten, vgl. Beızke, Uneilsanmerkung. SJZ 1948, Sp. 263: OGHZ 1, 35 (361.) v. 11.4. 1949.

76 Diesen Zusammenhang zur Identizüstheone (vgl. Fn. 10) betont Wengler, (Fn. 72), 5. 67 I.

77 Mituc $19 A^{8}$ gab es un der bruschen Zone unter den Landgenchuspräsidenten und Landgerichusdirekıo. ren $80-90 \%$ ehernalige Mieglieder der NSDAP, vgl. Stolle1s, (Fo. 70), S. 395. Zur Renazilizıerung der Justiz vgl auch Wenzlau. (Fn, y1), S, 94 If.

78 In der Recheslchre: Roemer, Von den Grenzen und Anunomen des Reclies, SJZ 1946, S. 9; Kisselbach, Zwes Prableme aus dem Gescis Nr,10 des Konirollrats, MDR 1947, S. 2. Weicere Nachweise bei Schumacher, (Fn, 2). S. y fl.: a. M. nur Jahreiß. Plidoyer im Nurnberget Prozeß v. 4. 7. 1946, $1 \mathrm{n}$ : Der Prozeß gegen dic Haupekriegsverbrecher vor dem intematsonalen Mihtërgenclitshof, Bd. XVII, Numbcrg 1948. S. $499(500)$.

In der Rechesprechung: $\Lambda$ G Wicsbaden 13. 11.1945, SJZ 1946, S. 36; OLG Frankfure 12.8. 1947, SJZ 1947, Sp. 622 (627). Weitere Nachweise bes Langner, Der Gedanke des Narurreches sell Weimar und in der Rechesprechung der Bundesrepublik, Bonn 1989, S. 93 ff.; а. М. и den veröffentlichecn Uncilen nut OLG Hamburg 18.6.1947, SJZ 1948, Sp. $35(36)$.

79 BGHSt 3, 357 (362 r.) v. 19.12, 1952; ebenso BGHSt 2, 234 (238) v. 29.1.1952; 2, 173 (177) v. 12. 2.1952. 
Auch die Unerträglichkeitsthese Radbruchs wird vom BGH und vom Bundesverlassungsgericht übernommen. ${ }^{\text {so }}$

bb) Neue Prufungskriterien

Im Gegensatz zu dicsen Bekenntnissen entwickelten Rechtslehre und Rechtsprechung ab Mirte 1947 in der täglichen Praxis, d. h., wenn konkres nach dem "rypisch

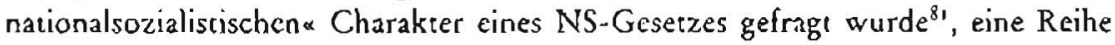
von Prüfungskrizerien, die der Verleugnungs- und der Unerträglichkeitsthese diametral entgegenstehen.

(1) Der objektıve Wonlaut emes Gesetzes

Die Frage, ob zur Ermicrlung des rypisch nationalsozialistischen Gehalts eines NSGeserzes die Morive des Geserzgebers, der politische Zweck und die Enrstehungsgeschichte dieses Gesetzes oder allcin der klarc Sinn des Worlauts und der objekrive Inhalt entscheidend sein soliten, wurde 1946 bis Mitte 1947 anders beantwortec als ab Mitte 1947. In der ersten Phase wurdc in Übereinscimmung mir Radbruchs Verleugnungsthese das Gesecz im nlichre der nationalsozialistischen Weluanschauung und ihrer Ziele $\alpha^{8_{2}}$ betrachtet. Entscheidend waren dic "seinerzeitigen Motive ${ }^{8_{3}}$ und der Makel der ursprünglichen Zweckbestimmung des Geserzes. ${ }^{84}$

Der objektive Wortlaut der Norm war gerade nicht maßgeblich:

"[Die Verordnung] beruht gemä̊ ihrem Inhalt auf typisch nationalsozialistischem Gedankengut, was zwar aus ihrem Wortaut und ihrer formellen Gestalung alkein niche ohne weiteres erkennbar ist, aus ihrer Entstehungsgeschichte und ihrem Zweck jedoch mit Sicherheit geschlossen rerden kann. ${ }^{85}$

Aufgrund dieser Prülungskriterien crkJärı die Rechrsprechung der ersten Phase z. B. den $\$$ is95a BGB in der Fassung vom 12.4. 1938 (wonach der Staatsanwalt neben den Eiterm die Möglichkeit hatze, im öffentlichen Inceresse die Ehelichkeic eines Kindes zeitlich unbegrenzt anzufechten) wegen sciner expliziten rassenpolitischen Ziclsetzung, wie sie in der Begründung des Gescrzes zum Ausdruck komme ${ }^{86}$, für

Dicsc Kernbereschstieone gile allerdings nach Ansıche des BGH nuc für dic materielle Geitung des Rechts. Für soin formelles Zustandekornmen ward jedes übergesetzliche Rechr verneint:

-Dic formelle Gulugkeit einer Rechisnorm - dic Fragc, ob eın Gesciz oder cine Rechtsverordnung veriassungsmäßig zustande gekommen sst - kann nur nacli Staalsrecht beurccift werden, das zur Zen dieses Zuscandekommens gilt oder gegolen hat... Die gegenteilige Auffassung.... (dic) dic Gülugken von Rechesnormen deshalb vemeinen will, weil sıe nach nauonalsozialisesschen Grundsäizen zustandegekommen seien, (aurde) au dem unmöglichen Ergebnis führen, daß nahezu dic ganx.e Geselzgebung der nationalsozsalistischen Zilt rechesuniurksim ivare.... Es Ist aneikannten Rechts, daß cinc gelungenc Revolution neues Verfassungsreche und neuc Gescizgeber schafit, die ncues formalrechulich güluges Rechu setzen können.* BGHZ s, 76 (94 ff.) v. 8. 2. 1952.

Diese Auslührungen begründen den bautosen Wiedereinsicg in das NS-Reclue. Nor vom 19s2 herrschenden Standpunki ener angestribien (Reches-)Konunuicai sielle sich die Rechesunwirksamkell nahezu der ganzen natıonalsozualisuschen Gescuggebung als nunnogliches Ergebnis dar.

so BGHSt 2, $234(238)$ v. 29.1.1952; BVeriGE 3, $225(232)$ v. 18.12.1953; ähnlich BVer\{GE 6, 132 (198)sog. Gesiapo-BeschluB.

81 Eine Liste der NS-Geselze, deren Fongcltung diskuetert wurde bea Stolless, (Fn. 70), S. 401 .

82 Das Numberger Jurstenureil, z.l. nach Stemiger, Lyszczynsk, Fall 3. Das Uneil im Jurıstenprozeß, (Ost-)Berlin 1969. S. go.

$9_{3}$ Buchwald, Gerechtes Recht, 2. Aufl., Weimar 1947, S. 27; Nathan, Uneilsanmerkung, NI 1947, S. 249; Wesss, Urteilsanmerkung. Ny 1947, S. 104.

84 Nalhan, Ureilsanmerkung. NJ 1947. S. 1631.; OLG Gera 30. 10.1946. NJ 1947. S.63.

8s LG Konstanz (Tillessen-Ureil) 28.2. 1947, SJZ 1947, Sp. 143 .

86 .JDic Befristung der Ánlecheung isr) mit heurjger Rechisauffassung unvereinbar. Dics wird bisondees deullich dann, wonn sich erst nach Ablaul der Anfecheungsfnst herausstelle, daß das Kind von einem Manne suamm?, der ener anderen Rasse als der Ehemann angehör. A ber auch in weniger keassen Fallen 2wingt dit Bcdcutung, dic nach hcutıger Anschauung dex blutsmäßigen Abstammung eınes Menschen zukommı dazu, die Anfechtung... stets zu ermöglichen.u (Begrundung zu dem Gesetz. über dic Xnderung und Erginzung familienrechtlicher Vorschiviten und uber die Rechessecllung der Starcenlosen vom 12.4.1938 (RGBI. I, S. 380) in: Deutsche Justiz 1938, S.619). 
ntypisch nationalsozialiscisch * und deswegen unanwendbar. ${ }^{87}$ Ebenso wurde 1946 auch die Weitergeltung des $\$ 640 \mathrm{StPO}$, der es ermöglichte, die rassenmäßige Abstammung eines Kindes festzustellen, wegen seiner noffensichtlichen Beziehung zur nationalsozialistischen Rassegeseczgebung* verneint. ${ }^{88}$

Ab Anfang/Mitte 1947 meinten Rechtsprechung und Rechtslebre plötzlich, aus Art. III Ziff. 6 MilRegG ı, wonach die Auslegung eines NS-Geserzes an dem "klaren Sinn des Wortlauts z zu orientieren ist, herleiten zu können, daß der Wille und die Motive des Geserzgebers und der damalige Zweck des Gesetzes bei der Frage nach der Weicergeltung eines Gesetzes nicht zu berücksichtigen seicn:

„[Djer Umstand, daß politische Moive bei der Neufassung einer Rechtsnorm von crhablichem Einfluß waren, verleith für sich allein dieser noch nicht den Charakier cincs nationalsozialistischen Geserzes. Ebensowenig ist dic Tatsache entscheidend, daß eine Rechtsnorm in nationalsozialistischer Zeit zu polinschen Zwecken mißbraucht [!] wurde, oder daß sie von vornhereın zu dicsem Zweck geschaffen wurde, zu politischen Zwecken mißbrauch zu werden. ... Maßgebend für die Beurteilung ist der objektive Inhalt. Denn Geserze führen nach ihrem Eslaß ein Eigenleben. «"

Nach einer solchen Reinigung der NS-Geserze von jeder politischen Zielsetzung und von jeder die reine Norm »mißbrauchenden « Anwendung stehe auch $\$$ Ig9sa BGB im demokracischen Staat niches mehr entgegen.

„Der Umstand allein, daB $\$ 1595 a$ BGB nationalsozialistischen Gedankengängen scincn Ursprung verdankı, kann jedoch diese Vorsclorift nicht unanwendbar machen.4\$

Das Prüfungskriterium objcktiver Worthaut negiert das oben entwickclte Prüfungsschema für gesetzliches Unrecht in drei Punkeen: Die in der Verleugnungsthese berücksichtigten Mocive des Gesctzgebers bleiben unberücksichtigt, die Frage nach der Legitimität des Geserzgebers wird überhaupt sicht gescellt, und indem ein "reines Geserz* angenommen wird, das nur durch die Rechtsprechung "mißbraucht* wurde, wird dic Praxis des Unrechts-Sraates, das Zusammenspiel von diskriminierender Gesezzgebung und willkürlicher Gesetzesanwendung, verkannt.

\section{(2) Bereinigende Auslegung}

Ist entscheidend für den nationalsozialistischen Charakter cincs Gesetzes allein dessen sachlicher, objekciver Inhalt, werden Formulierungen wie „Schutz des Volkcs « $(\$ 359$ StPO) oder "gesundes Volksempfinden ( $(\$ 240$ Abs. 2, 253 Abs. 2 StGB) zu den Gesetzen bloß äußerlicher „Phrascologie "', zu bloßen wnationalsozialistischen Schlagworten " ${ }^{92}$, zu eincr "nacionalsozialistischen Verbrämung eincs richrigen Rechtssatzes \&"s die die Norm noch nichr zum typisch nationalsozialistischen Gesetz stempeln. Vielmehr seien, um den richtigen, auch im demokratischen Staat anwendbaren Rechtssatz aus dieser "Verbrämung herauszuschälen, diese Schlagworte einfach durch wobjektive Betrachtungsmaßstäbe" zu ersetzen und das

87 Nathan, Uneilsanmerkung. NJ 1948, S. s1 f, OLG Halle :2. 11, 1946 . DRZ 1948. S. 99.

88 OLG Müchen S.11. 1946, SJZ 1947, Sp. 33: OLG Cclle 28. s. 1946, SJZ 1946. S. I \$4 min zust. Anm. Rosenberg, S]Z 1946, S. 155; OLG Seuregarn 7. 8. 1946; OLG Köln 4. 7. 1946; anders nur OLG Hamm 22. 5. 1946, SJZ 1946.S. 154 .

89 OLG Neuscade 4.8.1949, MDR 1949, S. 702 ; c benso KG Berlin 1.8. 1947, SIZ 1948, Sp. 262 (26,); KG Berlin 22.10.1947. JR 1948. S.165: OLG Hamburg 30.7.1947, MDR 1948, S. 26: OLG Kasscl

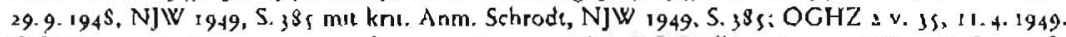

90 OGHZ 2. 31 (37) ‥11.4. 1949; ebenso zu \$ 15951 BGB, KG Berlin 1.8. 1947. SJZ 19.98. Sp. 242 f., OLG Kirsel 29. 8. 1948. NJW' 1949, S. 385; Guggumos, Urteilsanmerkung, NJW 1947/48. S. 388 ; ders. Dic Anfechung der Elsclichkett durch den Staztsanwalt, SJZ 1948, Sp. 247 f.: Beitzke, Uncilsanmerkung, (Fn. 7s). Sp. 26)

91 Guggumos, Uncilsanmerkung, NJW 1947/45, S. 3\$8.

92 OLG Neustodt 4. 8. 1949, MDR 1949, S. 702; OLG Koblenx 24.4. 1947, SJZ 1947, 5p. 441.

93 OLG Dresden 14.6.1946, DRZ 1946, S. 158. 
Gesecz einer rechesstaatlichen Auslegung zu unterstellen. Schon erhält man ein rechtsstaatliches Geserz!

"Die Ausdruckswcise rum Schuix des Volkese stelle allerdings cinen ausgesprochen narionalsozialistischen Begriff dar. Eine Rechesnom wird jedoch niche schon dadurch unanwendbar, daß in ihr en nationalsozialistisches Schlagwort enthaleen ist. Auch hier ist der sachliche Inhalt der Rechesnorm entscheidend und das bei objekuver, politisch unbecinflußter, rechisstaadjcher Geserzesauslegung erziele Ergebnis. Hierbei mussen Begrific wic schutz dis Volkese

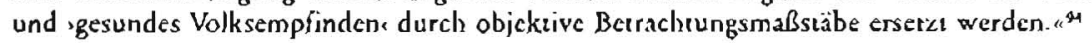

Damit ist die Anwendbarkeit der $\$ 240$ und $2 \$ 3$ StGB bejaht

"mit der Einschränkung, daß an die Stclle des Bcgriffs 'gcsundes Volksempfindenc der Begriff 'gute Sitene zu treten hat. $\alpha^{\text {ys }}$

Bis Ende 1946/Anfang 1947 war sich die Rechrsprechung hingegen weitgehend einig, daß nach Art.III Ziff.7 MilRegG 1, der die Bescrafung nach gesundem Volksempfinden verbot, auch die Anwcnabarkeit der $\$ 240$ und $253 \mathrm{StGB}$ ent(ä)lt, da der Begriff igesundes Volkesempfindenc das ganze Gesetz prägte.

- Alle Strafgesetze, die den Begriff des gesunden Volkscmplindens verwenden und dami den Anwendungsbercich des Strafgesetzes umschrciben, [haben] die Geliung verloren. "so

(3) Das nationalsozialistische Gesetz als Ergebnis einer normalen Rechtsentwicklung Isc das NS-Gesecz so durch die Eliminierung der ihnen zugrundeliegenden (rassen-) politischen Motive sentnazifiziere, und ist durch die Erserzung der nationalsozialisuischen Schlagworte durch neuc Begriffe nachgewiesen, daß das Gesecz einer wobjektiven Geseczesausiegung aus dem Geiste des rechtsstaatlichen Denkens",97 einer Füllung "der gegebcnen geseczlichen Tarbestände mic neuem Inbalt (98 $^{8}$ zugänglich ish erscheinc das Gescrz als Ergebnis weiner einwandireien Rechrsentwicklung ${ }^{\circ}, 99$

"Die meisten Richter machen es sich zuch heute wiederum sehr leicht. Sie lesen aus der amtlichen Begründung der natsoz. Gesetze oder aus der Begründung oberstrichterlicher Entschcidungen der Nazizeit rypisch natsoz. Gedankengut heraus und verncinen schon deshalb die weitere Gültigkeit eines natsoz. Gesetzes... Nur wenige Richrer besinnen sich wie chedem auf das wesendiche und versuchen derngemäß, in geeigneren Fällen nachzuweisen, daß das während der NS-Zeit erlassene Gesetz... trotz der nationalsozialistischen Phraseologie der rarsächlichen Enrwicklung der Dinge gefolgt, somit als Ergcbuis einer normalen Rechtsentwicklung, die es ja auch in der Nazizeit gegeben hat, anzusehen und daher weiterhin anwendbar und brauchbar ist. $x^{100}$

Das Abstellen auf den objekiven Wortlauz, die bereinigende Auslcgung und die Unterstellung der NS-Gesetze unter einen rechtsstaatlich-rationalen Rechtsbegriff und einer entsprechenden Gesetzesauslegung ermöglichte, dic NS-Gesecze als snormales Abweichen des 'Seinse von dem rechtlichen ,Sollen zu bewältigen $\alpha,{ }^{101}$ ohne sich die fundamentale Differenz zwischen einem rechtsstaatlichen und dem nationalsozialistischen Recht vergegenwärtigen zu müssen. Die genannten Prüfungskriterien erweisen sich damir als Grundlage der Konstruktion einer Kontinuität des

94 OLG Neustade 4.8. 1949, MDR 1949, S. 702; cbenso OLG P\{alz 8. 1, 1947, DRZ 1947, S. 235.

9) OLG Kablenz 21,4. 1947. SJZ 1947, Sp.441; Nicthammer, Urecilsanmerkung, DRZ 1946, S.61.

96 OLG Kablenx, cbd.; Nicthammer, cbd.; OGH 7.6. 1949, MDR 1949, S. j02; cbenso OLG Dorrmund 16. 4. 1946, SJZ 1946, S. $120:$ KG Berlin 10.7.:9.6, NJ 1947, S. 40 mil zustummender Anm. Werss, NJ 1947, S. 4x; OLG frciburg 5.6.1946, DRZ 1946, S.61.

97 OLG Neustadk 4.8.1949, MDR 1949, S. 702.

98 Weiss, Das Geserz uber die Kassation recheskräfuger Uncile in Stralsachen, NJ 1947, S. 213.

99 Ledig. Zum Kontrollearsgesetz 10, NJ 1948, S. 188 (190): Habcl, Ehelichkitusanicchtung durch den Staacsanwale, JR 1948, S. 176 .

100 Guggumos, Ureilsanmerkung, NJW $1947 / 48,5.388$

101 Kim, (Fn, J), S. 6 . 
Rechos über 1945 hinaus. Vor dem Hintergrund der durch die alliierren Geserzgebung eingeleiteten Entlegitimierung der NS-Rechtsordnung sind sie als Formen des lautlosen Wiedereinstiegs ${ }^{\text {Tox }}$ in das NS-Rechtssystem zu bezeichnen.

(4) Die Braucbbarkeit eines nationalsozlalistuschen Gesetzes in nexen Stant

Diese Überordnung der Rechrskontinuität über die Entlegirimierung der NSRechtsorónung dokumentiert besonders deutlich ein weiteres Prüfungskriterium. Ein NS-Gesetz sollte auch dann weitergelten, wenn es nauch unter den heurigen Verhältnissen inhaltlich einem Bedürfnis der Rechtspflege entspricht. $\alpha^{105}$

Auch deswegen sollte z. B. $\$ 1595$ a BGB weitergelten. Denn auch heute gäbe es

sein öffentliches Interesse an der Anfochtung. Fällc, in denen ein uneheliches Kind als ehelich behandelı wird, erzeugen of Unruhe in der Öffentichkeit. ${ }^{104}$

Auch die Kriegswirtschaftsverordnung könne nicht einfach außer Krift gesetzt sverden, da ihr Fortall muntragbare Konscquenzen a für dje Versorgungslage der Bevölkerung mit sich bringen würde. Deswegen sei es

wsachlich geboten, daß man zunächse die inhalelich unentbehrlichen Gesetze aus der Nazizerr einfach bestchen läßr. $a^{103}$

Die Frage nach der Brauchbarkeit eines NS-Gesetzes im neuen Staat kehre die Frage nach dem cypisch nationalsozialistischen Gesctz bzw. nach dem gesetzlichen Unreche und dessen (Weiter-) Geltung um. Es wird nicht gef́rage, ob ein Gesecz typisch nationalsozialistischen Charakter trägt und deswegen kcine Geltung hat, sondern es wird die koncinuirätsichernde Frage gestellt, ob der neue Staat das Gesecz brauchr, und daraus gefolgert, daß es dann nicht typisch nationalsozialistisch sein könne.

(5) Auch ein Rechtsstaat hat solche Gesetze

Das Vorliegen eines typisch nationalsozialistischen Gesetzes wurde weiter dann verneint, wenn sich eine dem NS-Gesecz encsprechende Regelung auch in demokratischen Rechtsstaaten findet. Dieses Prüfungskriterium wurde schon im Nürnberger Juristcnurceil angewendet. Dort heißt cs zur sog. Gewohnheitsverbrecherverordnung:

"Was die Bestrafung von Gewohnheitsverbrechern bctriff, sa glauben wir, Ist die Antworı klar. In vielen zivilisierten Staarcn verlangen geseczliche Bestimmungen, daß die Gerichce lebenslängliche Frciheitsstraicn wcgen Verbrechen verhängen, wenn bewiesen Ist, daß drei oder mehr Verbrechensbesırafungen vorgclegen haben. Wir können niche in einem Atemzug sagen, daß lebenslängliche Haft für Gewohnheitsverbrecher einc heilsame und vernunfrigc Bestrafung in Amerika in Friedenszecten Ist, daß aber die Verhängung der Todesstrale in Deutschland ein Verbrechen gegen die Menschlichkeıt war, als die Natıon unter dem Druck des Kricges stand. ${ }^{100}$

Dieser Vergleich zum Rechtsstaat wurde von der Rechrslehre und der Rechtsprechung ab 1948, vor allem bei der Prüfung der sogenannten Rundfunkverordnung, ${ }^{107}$ der Kriegssonderstrafrechtsverordnung, ${ }^{108}$ den Bescimmungen über Hoch- und

102 Kirn, (lin. 1). S. 64 spricht von "Formen des gerauschlosen Aussteıgens aus der NS-Verfassung". Doch auf dem Hintergrund der Unterbrechung der Recheskonesnustät durch dic alliierte Gesetzgebung ist besser von einem Wiedereinsuig al sprechen.

103 KG Berlin 22.10.1947, JR 1948, S. 165.

$104 \mathrm{OCHHZ}$, js (39) v. 11,4.1949; ebenso Beitzke, (Fn. 75), S. 264.

105 OLG Dresden 14.6.1946, DRZ 1946، S. 158: OL,G Kiel 7.2.1946. SJZ 1946, S. 118: OGHZ 2, 2s9 (263).

106 Das Nürnberger Junstenureil, zit. nach Ostendor, Vcen, Das Nürnberger Jurnstenuneil, Frankfur a. M. 1585, S. $17 \%$.

107 Vecordnung über außerordendiche Rundfunkmaßnahmen v. 1.9. 1939, RGBI. I, S. 1683.

108 Verordinung uber das Sondersiralrecht im Knege und bei besonderem Einsacz v. 17.8.1938, RGBI. 1939 I. S. 1455 
Landesverrat und der Standgerichtsverordnung ${ }^{109}$ aufgegriffen. ${ }^{10}$ Durch diese nkriegsrechtlichen Bestimmungen" habe der NS-Staat nur dem ihm - wie jedem (Rechts-)Szaat - zuszehenden Anspruch auf Schucz seines Bestandes und seiner staaclichen Organe normiert. Deswegen könnten diese Normen keine typisch nationalsozialisrischen Gesetze, kein gesetzliches Unrechr sein.

„[Dic Straikammer schließt ssch der von Radbruch verretenen Ansicht an, wonach] nur solche ordnungsgemäß verkündeten Gesetze und Vorordnungen des Hiclerstazres rechisungültig waren, die ihrem Inhalt nach klar gegen die Grundsätze der Gerechtigkci̊... verstoßen... Von den rein kriegsrechtlichen Bestimmungen läßt sich ein solcher Blanketecharakter niche behaupıcn, mögen sic auch der Aufrechterhaleung des inneren Regimes zurvilen dienen; denn ilır hauprsächliches Schutzobjekt ist - biervon unabhängig - dic Souveranitàt des Staates als solchem. ${ }^{\prime \prime \prime}$

Dicser Vergleich zum rechrsstaaclichen (Staatsschurz-)Gesetz verkennt die für die Radbruchsche Formel als essentiell herausgearbeitcte Berücksichtigung des Zusammenhanges des einzelnen Gesetzes zum gesamien NS-Rechissystem. Er ignoriert die Einbertung z. B. der Kriegssonderstrafrechtsverordnung in die Praxis des Unrechis-Staates, wodurch diese nicht dem Schutz des Staates, sondern der Vernichtung Andersdenkender diente. Auf die Unerträglichkeit der mit diesem Vergleich zum Rechtsstaac cinhcrgehenden Aufwertung der N5-Gesetze zum rechisstaatlichen Recht und des NS-Staates zum im Notstande belindlichen Rechtsstaat wies der OGH mehrfach mit scharfen Worten hin:

"Die vergletchenden Hinweise des Schwurgerichts auf gewisse Rechrscritwacklungen in England, der Schweiz und den Vercinigten Staten sind sachlich unzutroffend. Straftaten, die sich etwa ais, Welirkraftzerseczung kennzeichnen lassen, sind im Knege allerdings überall denkbar. Ein despotischer Stzat, der die Mcnschenrechte scinen Zielen bedcnkenlos unterordnet, versteht und benuizt den Begriff der Wehrkraftzerseczung aber anders als ein Rechisstaal... [E]nglische Bürger [werden nicht] wegen zwcifelhafter Äußerungen im Familienkreise über dic Recheslage wollkistlicher Polizciverfolgung und einer weithin getenkten Sonderjusiz, grausam harten Strafen und Konzentrationslagermethoden [ausgesctzt]... Nicht auf dic erwaige Strafbarkeit arger Quentreibcresen nach rcchisstaatlichen, gerechi gehandhabten Grundsätzen kommt es an, sondern aus die grausame, willkürliche sAusmerzunge Andersdenkender oder auch nur Zwcifelnder, die der natronalsozialisusche Stane derart zum Grundsatz erhoben hatte, daß sich cin Vergleich nsit rechtssiatlich gesinnten Völkern verbictet... Diesen

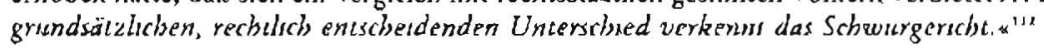

Der deurliche Umschwung der Prüfungskriterien zwischen Ende 1946 und Anfang 1947 fällt zusammen mit der Änderung des gesellschafrlichen Gesamtklimas von versuchtem Neubeginn zur Resauration." Diese zcitliche Parallelität zcigt, daß dic Bcantwortung der Frage nach dem typisch nationalsozialistischen Geserz mit diesem Wandel, insbesondere mit dem Vorverständnis der ab 1947 verstärkc in die Justiz eindringenden NS-Juriscen zusammenhängt." "4

"Jede dieser Enıscheidungen [ob eın typisch nationalsozialistisches Gesetz vorliegt], barg en Stück Diagnose des Nationalsozialismus und zuglesch ein Stück der . Moralitäk des nachträglichen Umgangs mit dicser Vergangenlicit. ""s

Und wie sah diesc „Moralität « des nachträglichen Umgangs aus?

109 Verordnung über dic Errichtung von Seandgerichten v. 15. 2. 1945, RBCI. I, S. 30.

io Feldmann, Das Verbrechen gegen die Menschlichkcit, Essen 1948. S. 51: LG IVorden 19. 2, 1948, in: Rüter (Hg), Justzz und NS-Verbrechen. Sammlung deurscher Strafureeile ivegen nauonalso'sulistuscher Tötungsverbrechen 1945-1966, Bde I-X'XII, Anstuedan) 1968-1981, Bd. II, S. 235 (275 (.). LG A achaifenburg 6.12.1948, 17: Rütcr, cldd, Bd. II7, S.62s $(649)$.

11 LG Weiden, ebd.

112 OGHSe 1, 38 (386f.) v. 20.4 1949; áhnlich OGHSt 2, 17 (18) ะ. 10.5. 1949:1, 219 (241) v. 4.1. 1949.

11) Vigl. Fn. 10 .

114 Siolleis, (Fn. 70), S. 398, 400

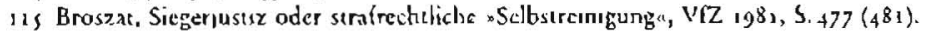


Die von der Rechtsprechung und Rechtslehre ab 1947 entwickelten Prüfungsteriterien führten zu einem nentnazifiziertena nationalsozialistischen Recht. Dabei bedeutet Entnazifizierung nicht die völlige Bescitigung des NS-Rechts, sondern die innerliche Reinigung, das Reinwaschen ${ }^{116}$ des NS-Gesetzes durch dessen Rcduzierung auf einen angeblich rechtsstaatlichen, objektiven Wortaut. Das Ergebnis ist ein "purifiziertes, narionalsozialistisches Recht ${ }^{1}$ " dic Legitimierung der Rechtsordnung des NS-Staaces.

\section{Die Auseinandersetzung um das Kontrollratsgesetz Nr. 10}

\section{Das Verbälenis von KRG 10 und deutschem Strafrecht}

In Art. III Ziff. rd KRG ro war die Möglichkeit vorgesehen, daß die Besatzungsbehörden für

mdic Abureeilung yon Verbrechen, die deursche Staatsbürger oder Staatsangchorige gegen andere deursche Staatsbürger odcr Staztsangchörige oder gegen Sta1tenlose begangen habenж, deursche Gcrichte für zuständig erklürcn.

Im Gegensatz zur amerikanischen Zone, die eine solche Ermächuigung nicht erreilte ${ }^{118}$, wurde in der französischen Zone mit einer generellen Ermächtigung ${ }^{19}$ und in der britischen Zone mit der Verordnung Nr. $47^{120}$ von dieser Möglichkeit Gebrauch gemachr. Diese Verordnung bestimme: Wenn ein Verbrechen gegen die Menschlichkeit

wauch als Verbrechen nach den gelecnden deurschen Gescezen anzusehen [ist], so kann gegen den Angeklagten Alternativklage erhoben werden. ${ }^{121}$

Was mit Alternativklage gemeint war, war in Rechrsprechung und Recheslehre umstritten. Nach der h. M. in der Rechrsprechung bedeutete Alternativklagc ein Entwcder-Oder, so daß ein Urteil nur auf KRG 10 oder nur auf dcutsches Strafrecht gestülzt werden könne. ${ }^{12 \alpha}$ Dieser Meinung, dic darauf zielce, das KRG 10 zu

116 Vgl parallel dozu den Wandel des Entnazifizaerungsbegriffs bei der personellen Eninaxifizicrung. Schon Ende 1946 konnec die Entnazifizierung in Deutschland nur noch als sw whte ivaslung a bezeiclinct werden, so Her, The Fiasco of Denazificauon in Germany, in: Policical Science Quancrly, Bd.63 $(1948)$, S. $569(573)$.

117 Grünwald, (Fn. 24), S. 16.

118 Nur aul besunderen Anirag crlolgte eine Freigabe des KRG 10, sowcie gleichzciblg deutsches Recht verlesze war, vgl. Lange, Zum Dcnunzianeenproblem, SJZ 1948. Sp. 302.

Sutudessen erging in den Ländern der amerikanischen Zone ein von deutschen Gerichten anzuivenden-

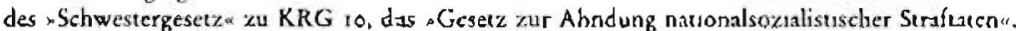

Bayem: 31. 5. 1946, GVBI., S. 182; Bremer: 27.6.1947, GBI., S. 83: Hessen 29.5. 1946, GVBI. S. i 6 : Wurtumberg-Baden: 3i. S. 1946 , RegBl., S. 17 .

$119 \mathrm{Vgl}$, Broszat, (Fn. 115). S. 496.

120 30.8. 1946, Amtsbl, d. MilReg. Deuscht. Nr. 13, S. 306.

121 Diese allgememe Ermachugung wurde durch eunen Erlaß der Milizärregıerung v. 10.9. 19,6 (JMBI. NRW 1947, S. (0) eingeschränkt: Nach KRG 10 durften nur Verbrechen von Gefingnisrvärem, der Gesispo, der SS und der zivilen Polizeı gegen Insassen von Gefängnıssen, Konzcntmatıons- und Zwangsarbetslagern abgeurteilt werden. Später wurde die Anwendung des KRG 10 w'ieder auf andere Fallgruppen erwerter: a) auf Denunzıanten (Erlaf3 der MilReg. v. 21. 11, 1946, JMBI. NR W/ 1947, S. jo), b) auf Serilisationsfälle und auf Falle von polituscher und rassischer Verlolgung, soweut sic sich nichı gegen Juden aufgrund ihree Rcligıon oder Volkszugehöngkeıt nchtercn. (Erlaß der MilRcg. v. 20. 12, 1946. JMBI, NRIV, S. SI)

c) Dicsc Einschränkung entfiel durch eincn Erlaß vom 5.7.1947. (cbd.), so daß deutsche Gerıchtc nun errnächugi waren, alle Verbrechen nach KRG io abzuurcilen, sorvet sie an deutschen Staatsangehorigen oder Stizatenlosen begangen wurden.

d) Auch diese Einselyränkung auf deuesche Suatsangchönge und Stzzecnlose wurde durch das Gesetz, Nr. 13 der Allisireen Hohen Kommission am 25.1 ). 1949 aufgehoben (Amesbl. d. Alliieren Hahen Kammission, S. 54 ).

122 OLG Oldenburg 23.9.1947, Nds Rpfl. 194\$, S.17: OLG Hamburg 1.8.1947, SJZ 1948. Sp. 35: OLG 
umgehen, lag die Auffassung zugrunde, daß das KRG ro kein neuer, selbständiger Tatbestand, sondem nur eine Zusammenfassung deutscher Srafgesctze unter einem neuen Terminus sei ${ }^{123}$ und deswegen das deutsche Strafrecht in der Regel zur Ahndung von NS-Verbrechen ausreiche. ${ }^{124}$

Nach anderer Ansicht mußte das KRG ro neben dem StGB angewender werden, denn Alternativklage bedeute, daß sich zwar die Anklageschrift entweder auf KRG ro oder deutsches Strafrecht stützen könne, der Richter aber dic Tat nach allen möglichen rechtlichen Gesichtspunkten, also nach KRG 10 und nach dem StGB, zu würdigen habe. ${ }^{2 s}$

Für diese Ansicht spriche, daß KRG 10 zweifellos ein selbständiger, über das deursche Strafrecht hinausgehender Tarbesand ist. ${ }^{26}$ Das KRG 10 wurde gerade geschaffen, weil davon ausgegangen wurde, daß das StGB, welches jede Handlung zunächst als Einzeldelikt betrachtet, den kriminologischen Gehalt der NS-Verbrechen, den besonderen Unrechtsgehalt der massenhaften Häufung von Verbrechen des Staates nicht erfassen kann.'27

Ob der sich aus der Durchsicht der juristischen Zeitschriften von 1946-1950 ergebende Eindruck, daß diese zreire Auffassung von der Mehrheit der Rechrslehre vertreten wurdc, ${ }^{128}$ den wirklichen Mehrhcitsverhältnissen entspricht, ist fraglich. Denn die Milizärregierung griff durch cine gezicle Veröffentlichungspolitik in dic Diskussion cin. ${ }^{129} 130$

2. Die Einengung des Tatbestands von KRG 10

Eine weitere Form des Widerstands gegen das KRG ro bestand darin, die Alundung

Köln 14, 10. 1947, JR 1948, S. 53. In der Recheskchre: Hühnemörder, Rundschau, MDR 1947, S. 286; Meycr, Das Kantrollrarggeselz Nr. 10 in der Praxis der deutschen Siralgerichie, MDR 1947. S. 110 (III).

123 LG Erfur-Nordhausen 7.5 .1946 (Puufarken-Fall), in: Akıen des Zentedjustızamtes für die briusche Zonc, $Z_{21 / 1334}$ fol, 136, S. 7 (Bundcsarchiv). Zwcifel am Vorliegen enes selbständigen Tatbescands, Hodenberg, Zur Anwendung des Kontrollrakgesetzes Nr. 10 durch deutsche Genche. SJZ 1947. Sp.113 (117).

124 Daraus lertele enc zwette Anstche sogar dic Subsidiartic des KRG 10 ab, so Hodenberg. (Fn. 123), S. 116; OLG Oldenburg 20.1.1948, Nds. Rpfl. 1948, S. 68; LG Aunch 21.4.1948, m: Rürer (Fn. 110), Bd. II, S. 433: LG Götungen 12. 2. I951, MDR 1951, S. 11 2.

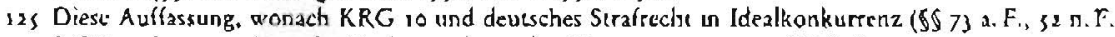
SIGB) stehen, wurde in der Rechlsprechung der Westzonen nur vom OLG Branschweig 29.11. 1947, SJZ 1947, Sp. 268 und vom OGH, OGHSt I, I (Lertsatz 3) v. 20.5.1948; 1, 39 (41) v. 20.7.1948 vertruten.

126 E. Schumid, Rexlitsgutachern uber Ar. IT Ic, f und s des Konrollratsgesetzes Nr. 10, in: Akeen des Zentrajustizamtes fur dic brilesche Zonc, Z $21 / 799$ (Bundesarchiv), S. iff.; Kraus, Kommenear zum Kontroliratsgesetz Nr. 10. Hamburg 1948, S. 7 f., Feldmann, (Fn. 1 10) S. 19 ff, Güdc, (Fn. 57), S. 1 14: Radbruch, Zur Diskussion...., (Fn. 3), Sp. 133; Wimmer, Die Bestralung von Humanizätsverbrechen und der Grundsatz onulla poena sine legew. SJZ 1947, Sp. 123 (126); Serucksberg, Zur Anwendung des Kontrollratxpesetzes Nr. 10, DRZ 1947. S. 277; OLG Kóln 14.10. 1947, JR 1948, S. 33.

127 Güdc, (Fn. 57), S. I 14; W/immer, (Fn. 126), Sp. 129.

Zum Versuch, scaudich organısıere Massinverbrechen mi den Teilnahmekonstrukuonen des SiGB zu crfassen: Roxin. Straftaten im Rahmen onganistercer Machtapparatc, GA 1963, S. 193 ff., Baumann, Beihille bet elgener voller Tarbescindsverwirklichung, NJW 1963, S. 561 ff.

$128 \mathrm{Vgl}$. Fn. 126 .

129 Broszat, (Fn. 115), S. 529, weist cinen Fall nach, wo die Veröffentlichung ones für dic MDR geplanten Anitels. der lür dic walılwerse Veruncilung nach KRG 10 oder nach $\mathrm{StGB}$ pladicrte, verhndern w'urde.

130 Nach eince vertretenen (Minder-)Manung ast das KRG 10 lex spezalis, d. h. bei der Ahndung von NSVerbrechen allein anzuwenden (Kraws, (Fn. 65), S. 76; L.G Konsianz 28. 2. 1947 (Tillessen-Urecil), SJZ 1947. Sp. 337 (342); OLG Tübungen 14. 3.1910, in: Rüter, (Fn. 120). Bd. V. S. 122). Das wird damit begründet, daß die Anwendung deutschen Strafrechts den Eundruck erweckt, als seien die NSVerbrechen das vercinzclee Abiveichen von einer sonst intaken Reches- und Staatsordnung gevesen. Denn das StGB ubaut aul der Vorausserizung auf, daß eine von der vollizichenden Gewale geachrete und behütete Rechtsordnung besteht und daß das Strafreche dazu dient, enen Angriff abzusehren, der sich gegen die solchermaßen geschütze Rechtsordnung richect. Die Handlungen der Angeklagren aber hielten dic von der vollzuchenden Gewalt eingeschlagene Richtung ein. Das Staatsoberhaupt hatce die besechende recheliche Ordnung... selbst zerstort، (OLG Tubungen, ebd.). 
von NS-Verbrechen durch die Enrwicklung zusätzlicher, nicht in KRG ro enthaltener Tarbestandsvoraussetzungen zu begrenzen. So wurde für die Bejahung des objekciven Tatbestands nicht für ausreichend gehalten, daß die Tat in Zusammenhang mit der Gewalt- und Willkürherrschaft des Nationalsozialismus stand, " " sondern der Täcer mußte gehandelt haben, um die NS-Herrschafr zu unterstürzen oder aufrechtzuerhalten. ${ }^{132}$ Zweicens sollre die Tat - obwohl das KRG ro Einzeltaten und Massenverbrechen für strafbar erklärt - kein Einzeldelikt sein dürfen, sondern müsse sich als Teilnahme an einem Massenverbrechen darstellen. ${ }^{33}$

Ab Mitte 1948 wurde von der Rechtsprechung und der Rechtslehre - vor allem bei den sogenannten Denunziacionsverbrechen - für den subjekriven Tatbestand gefordert, daß der Täter aus politischen, rassischen oder religiösen Gründen odcr aus unmenschlicher, verwerflicher Gesinnung gehandelt haben müsse, '14 anstart es - wie der OHG - ausreichen zu lassen, daß der Denunziant den Angezeigren den willkürlich handelnden Organen des NS-Staares auslieferte und sich des darin liegenden Angriffs auf das Opfer bewußt war. "s"

Diese Forderung zielte darauf, den sogcnannten Mitläufer zu exkulpieren. Freigesprochen wurde derjenige, der cinen anderen in der Überzeugung anzeigte, damit seine staatsbürgerlichen Pflichten zu erfüllen, ohne dabei explizit aus politischen Gründen zu handcln:

"Nicht jeder Denunziant als solcher ist sirafbar. Eine auf schuldloser Verkennung staatsbürgerlicher oder amtlichcr Pflichten und auf innerster, wenn auch verkehrter Überzeugung beruhende Denunziation seirens einer dadurch irregeleiteten Person wird niche als eine

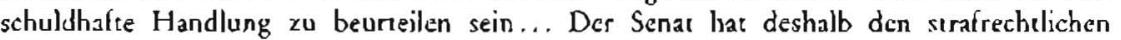
Schwerpunkt des Delikes der Denunziation in subjekuver Hinsicht in der Gesinnung des Denunziancen erblickt und für die Strapbarkeit erfordert, daß dic Denunziatıon einer verwerflichen, niedrigen Gesinnung cnesprungen sein muB, “' 36

Die mit der Forderung einer unmenschlichen Gesinnung einhergehendc Vergcistigung der Verletzung menschenrechelicher Kerngarantion verlegt die Strafbarkeit des Verbrechens gegen die Menschlichkeit von der objekriven Rechrsverleczung in das Innerc des Täters:

„Der Oberste Genchtshof verlangr, das Opfer müsse in seiner Menschenivuirde zuttefst getroffen scin. Würde ist Ausdruck einer Gesinnung. Sie wird nur durch eine verwerfliche Gesinnung ... verletzi $\alpha .{ }^{[3]}$

Damir unterliegt die Strafbarkeit von NS-Verbrechen dem restaurativen Bewußrsein der Zeir. Dic Verdrängung eigener Scluuld, dic Auffassung, nur eine kleine Clique sei für die narionalsozialiscischen Verbrechen verantwortlich gewesen ${ }^{18}$, während der

331 Der OGH wies dic Untergenches wiederhoir darauf hin, daß dies ausrechend sei. OGHSt 1, 6 ( 7 f.) v 25. 5. $1948 ; 1,11$ (Leissaz) v. 20.5.1948; 1, 45 (49) v. 27.7.1948; 1, 67 v. 17.8.1948; 1, 122 (123) v 16. 10. 1948;1, $167(168)$ v. 16.11, 1948.

132 Erdsıek, Ureei)sanmerkung, SJZ 194S,Sp. 39: OLG Oldenburg zit. nach OGHSt 1, 167 (168) v. 16. $11,1948$.

133 Endsiek, ebd.; Güde (Fn. 57), S. 11s; v. Weber, Das Verbrechen gegen dic Menschlichken in der Rechesprechung. MDR 1949, S. 261 (264): Radbuch. Uneilsanmerkung zum Tillessen-Urteil, SJZ 1947. Sp. 343 (344); Kraus, (Fn. 65), S.78; OLG Konstany. (Tillusscin-Uricil) 2.9. 1947, MDR 1947. S. 30 ; OLG Kicl, in: Rüter, (Fn. 110), Bd. III, S. 4061 .

134 Feldmann. (Fn. 110), S. 36 ff.; OLG Celle 15.9. 1948, Nds. Rpfl. 1948, S. 218 ; LG Hannover 1.7.1948, In: Rülcr, (Fn. 110), Bd. VI, S. 2\} z f.: LG Hildesheim 1\}. 12. 1949, in: Ruicer, (F1). 110), Bd. V. S. 664 ff., LG Hamburg 28.8.1950, in: Rúcr. (Fn. 110), Bd. VIl, S. 100 .

13) OGHSt I, 11 (16) v. 2.6.5.1948; ;, 60 (61) v. 17.8.1948; cbenso OLG Düssildorf 1A. 11. 1947. MDR 1948. S. 123 (124): OLG Braunschwe1g 28. 11.1947 , Nds. Rph. 1948, \$. 8 (s (2); LG Berlin 22.11. 1947, in: Ruier, (Fn, 110), Bd. 11, S. 192.

136 OLG H.mm 29.11.1947. MDR 1943, S. 94.

137 OLG Celle 15.8. 1948, Nds Rpfl. 1948, S. 218.

ij8 A. u. M. Miescherlich, Dic Unfähıgkeı zu travern. Mùnchen 1977. S. 27: M. Mitscherlich, Ennncrungsarbeit. Zur Psychoanalyse der Unfihigkeıt zu trauern. Frankfurt a. M. 1987; Pollock (Biarbeitcr), 
Durchschnitsdeutsche nur ein verführter Christr gewesen sei, fand Eingang in dic Rechtsprechung zum KRG 10:

-Der Angcklagte von W. seeht und stand stcis auf dem Boden cines glaubigen Christentums. ... Nach den Fesestellungen zur Personlichteir beider Angeklagien baben sic in ihrem Denken und Handeln in eınem mehr oder weniger offenen Gegensatz zu wesentlichen Grundzigen des Nationalsozialismus... gestanden und von ihrer Stelle aus gemensam versucht, auf $\mathrm{dem}$ Boden der damaligen politischen Wirklichtecir das von ihnen Jür Recht und richtig Eskannte durchzusctzen und so ihrem Volk und Staat zu dienen. [...]

Es ist nicht als crwiesen anzuschen, daB der Angeklagie von W. den M. aus politischen Gründen verfolgı hat. ${ }^{13 y}$

Solche Äußerungen zeigen, daß die Argumentation der Rechtslchre und der Rechtspraxis sich von der öffentlichen und massenhaften Derealisation der NS-Vergangenheit $^{\text {150 }}$ micht unterscheidet, dicse vielmehr in der juriscischen Argumentation ihren Fortgang findet. Im Widerstand gegen das KRG ro nimme die Schuldabwehr die -Gestalt juristischer Konstruktionen « an; sie lassen sich als "Unfahigkeir, in rechtichen Formen Trauerarbeit zu leisten ", charakterisieren. ${ }^{\prime+1}$

\section{Due Verletzung des Rückwirkungsverbous}

Schon im Dezcmber 1945, neun Tage vor dem Erlaß des KRG 10, bezog Lange in einem Gutachten Stellung gegen ein rück wirkendes Gesetz, weil

- das demokratische Rechr seinen Neuaufbau unmöglich mı der Verleızung scincs fundamenualsten Grundsatz[es] beginnen [durfe]. "'s

Dieses Argument wurde von E. Schmidt, Hodenberg und Feldmann außgegriffen. ${ }^{23}$ Es wurde verbunden mit der an die Alliierten adressierten Forderung, die Anwendung des KRG ro durch deutsche Geriche niche zu ermöglichen, ${ }^{14}$ und der Aufforderung an den deutschen Richter, zu prüfen, ob er dem KRG 10 nicht wegen der Verletzung des Rückwirkungsverbots die Anwendung versagen müsse. ${ }^{14} 4 \mathrm{Ho}$ denberg, der sich r 948 durch einen Arrikel in der Sondernummer der SJZ vom März 1947 an die Spitze der Opposition gegen das KRG 10 setzte, fügte ein wciteres Argument hinzu. Er meinte, daß die Argumentation der Verfechter des KRG ro, eine rückwirkende Bestrafung sei aus Gerechtigkeitsgründen notwendig, ${ }^{46}$ Rechtsstaatlichkeit und Rechtssicherheit wie in der NS-Zeit negiers.

"[Es] werde an die Stelle des den Richter bindenden Gesetzes - genau wie in der Nazizeit - das gesunde Rechisgefühl der Volksgemeinschaft geseczt. Es wird dabes im Ergebnis wiederum dieses Rechesgefülly der Volksgemeinschafi in Gegensazz gestelle zu dern durch das formelle Gesetz gevaahrleıstcien Schutz des Individuums, es würde also auch insowert der 193 s betonte,

Gruppenexpersment, in: Fronkfurter Bentrage zur Soziologie, Bd, z, hg. v. Adorno, Dirks. Frenklure 2. M. 1955, S. $308 \mathrm{HF}$.

13 LG Hamburg 28. 8. 1990, in: Ruiter. (Fn. 110), Bd. VII, S. 300 I., ïhnlich L.G Essen 9. 3. 1953, in: Rüzer, (Fn. I10), Bd. X. S. 594: LG Hanrover 1. 9. 1948, in: Ruter, Bd. VI, S. 237.

140 A. und M. Mischerlich, (Fn. 138), S. 14 .

141 Perels, (Fn. 8), S. 172.

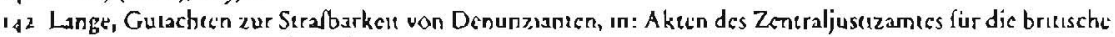
Zone, $Z_{22} / 1334$ (Bundesarchive), S. 2 .

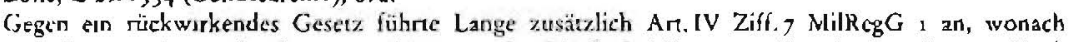
-Uncile nur erlassen, Strafen nur verhänge werden [dürfen], falls cin zur Zeil der Handlung in Kraft befindliches Gescez diese Handlung ausdrücklich für strafbar erklärt. ¿ Daraus eın Verbor zum Erlaß rückwirkender Geselze abzuleuten, sunmt mu dem Zweck des MilRegG i niche uibereun. Das MilRegG 1 wollte nur natronalsozialistische Vorschriften, die die riekwirkende Bestralung zuließen, authrben. Fis sollee damı kesın Grundsatz für dic künfuge allierte Gesetzgebung aufgessellt werden, vgl.

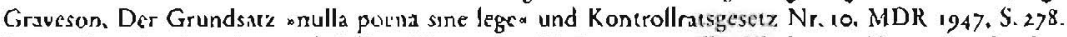
Das crgibi sich schon daraus, daß die Alliieren das KRG 10 mut "voller Klarheit und betonecr Absicluta Irotz des MilReyG I erließen, Bader, Unschau, DRZ 1946, S. 140 (141).

14) Schmidt, (Fn. 126), S. s if., Feldmann, (Fn. 1 10), S. 20 .; Hodenberg, (Fn. 123 \}), Sp. 119 f.

${ }_{144}$ Schmidi, (Fn. 126), S. 11, 16.

14) Hodenberg, (Fn. 12j), Sp. 19: Feldmann, (Fn. 110). S. 20 l.

${ }_{14} 6 \mathrm{Vgl}$. die Argumentation im folgenden 
damals neuc Grundsatz der Naxizeıt durchgeserzt, daß der Schutz der Volksgemeinschafı den unbedingten Vorrang vor den Belangen des Individuums... zu beanspruchen habe. "'th

Diese Argumentacion iss aus zwei Gründen unhaltbar. Indem die matcriclle Gerechrigkeit der Rechtssicherheit gegenübergestells wird, wird übersehen, daß hier die Herstellung der Gerechrigkcit gerade auch der Rechessicherhcit dient, weil sie die durch dic Nicht-Ahndung von NS-Verbrechen eingetretene Rechesunsicherheit beseitigt. Zweitens verkennt die Gleiclsstellung der ausnahmsweisen Durchbrechung des Rückwirkungsverbots durch das KRG to mit dem Recht des NS-Staates, daß das Rückwirkungsverbot im NS-Staat nicht nur für cincn Fall außer Kraíc gesctzt wurde, sondern die Rechtsunsicherheit zum generellen Prinzip crhoben wurde. ${ }^{18}$ Der Vergleich zivischen KRG 10 und NS-Rechtspraxis erhebt das NSReche in unerträglicher Weise zum cin wenig von der Norm abweichenden rechtsstaatlichen Recht, diffamiert das KRG ro als Ausfluß nationalsozialistischen Rechrsdenkens und verwische dadurch die Grenze zwischen Reches- und UnrechtsStaat.

In den juristischen Zeitschriften der Jahre $1946-1950$ ist nur ein Gerichtsurteil veröffentlicht, das die Anrvendung des KRG 10 wegen der Verletzung des Rückwirkungsverbors ablchnt. ${ }^{149}$ Nach Ansiche aller anderen Gerichte steht das Rückwirkungsverbot der Anwendung des KRG ro nicht entgegen.' so

Dieses Bild entspricht wohl nicht den wirklichen Mehrheitsverhältnissen, denn der Obcrstaatsanwalt in Konstanz, Güde, schreibt im April 1947: Die dcursche Rechtsprechung har "unverkennbar Hemmungen ", das KRG 10 wegen der Verlerzung des Rückwirkungsverbotes anzurvenden. 's' Erst im Scptember 1949, nachdem der OGH mehrfach ausgeführt hat, daß das Rückwirkungsverbot nicht verletzt ist, ${ }^{152}$ kann gemeldet werden:

-Die Verbindlichkeı des KRG ro steht, abgesehen von ganz vercinzelten, aus dem strafrechulichen Rückwirkungsverbot hergeleitcen Zwcifcln im Schriftum, in der deuscher Rechispraxis fest. "'s\}

Dic Auffassung, daß das Rückwirkungsverbot der Anwendung des KRG 10 nich entgegenstcht, kann sich auf eine Vielzahl von Argumenten stützen, die sich dadurch auszejchnen, daß sie das Rückwirkungsverbot zurückführen auf scine das Individuum schürzende, Gerechtigkeit und Rechtssicherheit intendierende Funktion und es cinbinden in die Lebre vom übergesetzlichen Rechr.

Nach einem vor allem von Radbrucl vertretenen Argument wirkt das KRG 10 gar nicht zurück, weil dic Taren, dic das KRG 10 für strafbar erklärt, schon zur Zeit ihrer Begehung - freilich in nichc positivierter Form - nach übergescuxlichem Recht Verbrechen waren. Dem KRG 10 komme nur deklaratorische, nicht rückwirkend

147 Hodenberg, (Fn. 123 ), Sp. 19 ; ebenso l.ange auf ciner serafrechelichen Arberrstagung. in: Akeen des Zentmljustszamtes für die britusche Zone Z 21/1314, \{ol. 140 (Bundesarchiv).

148 Diese Kritik an Hodenberg bo Wimmer, (Fn. 1>6), Sp. 126 u. Strucksberg, (F-n. 126 ), S. 278

149 LG Siegen 8. 5. 1947. MDR 1947, 5. 201. Aufgelıben von OLG Hamm 21.6. 1947, MDR 1947, 5. 20, Daraufhin numme das L.G Siegen von sesnen Bedenken Abstand, LG Siegen 9.9.1947. in: Rüucr, (Fn. 110), Bd.1, S. $663(668)$.

150 Das case-law-Arqumext verwenden: OLC Hamburg 18.5.1947, MDR 1947, S. 241 (242); OLG Braunschivelg 29.11. 1947, NJW/1947/48, S. 353; LG Freiburg 9.12. 1947, 1n: Ruter, Fn. 110), Bd. I,

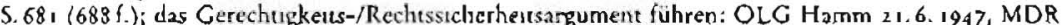
1947, S. 205: KG Berlin 12.2.1947, DRZ 1947. S. 334; OLG Koln 14-10. 1947. JR 1948. S. 53: OLG Braunschweig $24.11,1947$. NJW $1947 / 4 \times$. S. J13: das Funktionsargument benutzen: KG Berlin 17. 5. 1947, DRZ 1947. S. jos (305 (.). Zur Eintcilung dieser Argumente vgl. sm lolgenden.

15i Cide, (Fn. 57). S. Iu,

is 2 OGHSt 1, 1 (4) v. 4.5.1948: 2, 293 (297) v. 15.2. 3949: 2, 334 (335) v. 12.7.1949:2, 375 (378 f1.) v 21. 3.1950 .

153 Jagusch, Dus Verbrecken gegen dic Merischlichkeit in der Rechusprechung des Obersten Genclotshois für dic britusclic Zone, SJZ 1949, Sp. 620. 
surafbegründende Bedeutung zu. Die bestehende echische Verbotsnorm wird durch das KRG ro nur positiv-rechtlich festgeschrieben. 's4

Ein zweites, ebenfalls von Radbruch entwickelces, sogenanntes wcase-law-Argument « besagt, daß ein Widerspruch zwischen KRG 10 und dem Rückwirkungsverbot auch deshalb nichr bestehc, weil das Rückwirkungsverbot nur für kodifiziertes Recht, nicht für das case law oder common law des anglo-amerikanischen Rechtskreises gelte. Das Rückwirkungsverbor kann für Fallrecht, das sjudge-made-law a, wie das KRG so, das nur beispielhaúr bestimmte Handlungen für strafbar erklärt, das nur einen Leitsacz enthält, den die Justiz weiterzuverfolgen hac, 'ss gar nicht geiren, da es

nim Wescn des judge-made-law (liegt) zurückzuwirken: Der erste Fall einer neuen Rechisprechung fält notwendig unter ein Recht, das noch nicht bestand, als er sich verwirklichte. "ist

Nach anderer Ansicht verletzr das KRG ro zwar das Rückwirkungsverbor, doch ist scine Durchbrecliung vom Standpunkt der Gerechtigkeit und Rechtssicherheit ausnahmsweise geboten, weil eine große Zahl schwerer Verbrechen vorliegt, die aus politischen Gründen nicht bestraft wurden, und weil das Strafrechr der Begehungszeit zu ihrer Ahndung nicht ausreicht. Deswegen ist zur Wriederherstellung der Gerechrigkeit und der Rechtssicherheit dieses Pflichtversäumnis des NS-Staares im Wegc der Durchbrechung des Rückwirkungsverbotes nachzuholen. '37

"Nach der Auffassung aller sirlich empeindenden Menschen wurde schweres Unrecht begangen, dessen Bestrafung rechissiaatliche Phicht gewesen wäre. Die nachirägliche Heilung solcher PRicheversäumnisse durch rück wirkende Bestralung entspricht der Gerechigkeit. Das bedcutet auch keine Verletzung der Rechtssıcherheıt, sondern die Wicderherstellung ihrer Grundlage und Voraussetzung. Unrechtssicherung ist nichr die Aufgabe der Rechussicherheil.. '1 $^{8}$

Die Notwendigkeit der Durchbrechung des Rückwirkungsverbots läßt sich am überzeugendsten mit dessen Funktion begründen. Das Rückwirkungsverbor, das den Schutz des einzelnen Bürgers gegenüber willkürlicher Ausübung der Staatsgewalt bezweckt, ist historisch aufs engste verbunden mit dem Kampf des liberalen Bürgertums für den Rechtsstaat, für die Herrschaft des Gesetzes. 's9 Es ist also funkcional auf cine rechtsstaatliche Ordnung bezogen. Würde das Rückwirkungsverbot aus diesem Zusammenhang des Kamples um lndividualrechisschurz und Rechesstaatlichkeir gelöst und übertragen auf die Frage, ob die Verbrechen des Staates, seiner Organe und Träger nachträglich einer Ahndung zugeführø werden dürfen, würde das Schutzobjekt und damit die rechtsstaazliche Funktion des Rückwirkungsverbotes ins Gegenteil verkehrt. Es würde zum "Garanten der sankuionsfreien Willkür-Staarsgewalt des NS-Syscems " ${ }^{\text {to }}$ Die Schlußfolgerung liegr auf der Hand: Nur wenn das Rückwirkungsverbot für die Ahndung der Verbrechen des

Is4 Radbruch, (Fn, 1), S. 135 f.; ders., Geserz und Recht (Fn. 6), S. 1: Lange, (Fn. 118), Sp. 303: Bauer, (Fn. 56), S. 107; Güdc, (Fn. 57), S. I14; Wimmer, (Fn. 126), Sp. 127.

Is Radbrich. (Fn. 3). Sp. 13 3; v. Weber, Das Verbrechen gegen die Menschlichkeit in der Rechisprechung. MDR 19,19, S. 261 (263); Hodenberg, (['n. 123), Sp. 117 . Auf den Vorwurf Feldmanns, (Fn. 110), S. 22 , 74, das KRG 10 verlezze das Bestunmtheiesycbot, weil es nur beispiclhafi bestummte Handlungen für strabar erklïrt und keınen (est umnisscnen Tabestand enthik, soll hier nicht eingegangen werden, weil er in der Diskussion um das KRG io nur cinc uniergeordncte Rolle spielic. Der OGH mante, daß nder Fassung des Gesetzes in Verbindung mit dem klaren Sinn und Zweck an tundiutiger gesetzlicher Tatbexitand von hinreschender Besummtheit zu entnehmena 1st, OGHSi 1, 293 (297).

156 Radbruch، (Fn. 3). Sp. 134; s. 1. Kisselbach. (Fn. 78), S. 4; Meyer, (Fn. 121), S. 112; Wemer, Die crsten Entscheidungen des OGH z.um Kontrollratsgesetz Nr. 10. NJW 1949, S. 170. Anm.4.

157 Wimmer, (Fn. 126), So. 129 ff.; Graveson, (Fn. 142), S 280; Strucksbcrg, (Fn, 126), S. 279; Langc, D2s Koncrollraisgesciz Nr. 10 in Theonc und Praxis, DRZ 1948, S. 155.

158 OGHSt 1, I, (s) v. 8. 5. 1948:2, 375 (3801.) v. 21.3. 1950:2, 231 (232) v. $21.3 \cdot 1950$.

is9 Neumann, Dic Hereschafe.... (Fn. 27), S. 257.

160 Perels, (Fn. 8), S. 369. 
Unreches-Sraates durchbrochen wird, kann es seine ursprüngliche Funktion, die Staarsgewalt an Strafexzessen gegenüber den Bürgern zu hindern, auch wirklich errüllien. ${ }^{161}$ Dieses Argument finder in prägnanter Formulierung Eingang in die Rechtsprechung des $\mathrm{OGH}$ :

${ }^{\circ}$ Das Rücls wirkungsverbor gchör aber zu den Rechrsgrundsätzen, die dem Staarsabsolutismus im Kampl für dic Menschen- und Bürgerrechte abgenungen worden sind, um den Bürger gegen Stzatswillkür zu schützen. Daher empfängt es seinen Sinn. Es hieße aber dicsen Sinn ins Gcgenteil zu verkehren, wenn das Rückwirkungsverbor dazu dienen sollte, dic gerechce Sühne für solche Verbrechen zu vereiteln, die gerade in der Betärigung schrankenloser Stadeswillkiïr bestanden. Das Koncrollratsgesetz $\mathrm{Nr}, 10$ verstößz deshalb, auch wenn es auf vor seinem Inksafureten begangene Handlungen angewendet werden will, nicht gegen den dem Rückwirkungsverbor zugrundeliegenden Rechterigungsgrund. $\mu^{162}$

Die Durchsicht der juristischen Zeitschriften von 1946-1950 legt den Schluß nahe, daß dic Mehrheit der Juriscen den Argumenten von Radbrucl und Wimmer folgte. ${ }^{1{ }^{6} 3}$ Broszat, der auch unveröffentlichtes Material auswertece, komme dagegen zum Ergebnis, daß die Richter und Rechrsanwälte der britischen Zone in ihrer Mehrheit das KRG 10 wegen der Verletzung des Rückwirkungsverbores ablehnten. ${ }^{64}$ Diese Diskrepanz zwischen veröffentlichter und durchschnittlicher Meinung könnte wiederum mit dcr alliierten Veröffentlichungskontrolle zu erklären sein. Das Zentraljustizamt für die britische Zone wurde durch die britischen Besarzungsbehörden angewiesen, die Veröffentlichung von Artikeln, die sich ablehnend zum KRG to äußerten, zu verhindern. Gieichzeicig bemühte sich die britische Mililiärregierung um Publizierungsmöglichkeiten für Juristen, die den Artikel Hodenbergs kritisierten. $^{\text {ts }}$

\section{Statistische Aussagen zur Anwendung des Kontrollratsgesetzes Nr. 10 durch deutsche Gerichte}

Anband der Kriminalstatistiken der Länder Rheinland-Pfalz, Niedersachsen und Nordrhein-Westfalen ${ }^{168}$ lassen sich zwei Fragen beantworten:

- Inwieweit wirkten sich die vor allem in der Rechtslehre entwickelten Gcgenposicionen zum KRG 10 in der Rechtsprechung aus?

- Besteht ein Zusammenhang zwischen gesnmegesellschaftlicher Restauration und der Anwendung des KRG so durch die Gerichte?

161 Ebd.

162 OGHS1 2, 37s (380) v. 21.3.1950; sehr ihnlich Radbruch, Dic Emewerung.... (Fn. 6), S. 2; ders., (Fn. 3), Sp. 136.

163 Wemer, (Fr., is6), S. 170; Lange, Die Rechisprechung des Obersien Gerichishoís für die beulsche Zonc, SJZ 1948. Sp.655 (6 56$)$ : Jaghusch, (Fn. 153), Sp. 610; Strucksberg, (Fn. 126), S. 272; Kisselbach, (T.n. 78), S. 2

164 Braszal, (Fn.:15), S. \$2?

16 , Ebd.

166 Nur die Krımenalstatıstiken dicser Lander cnthalten annähernd vollständiges Zablenmaterial, so daß dic gemachıen Aussagen nxcht allgemeingültıg für alle Länder scın kónnın.

Einc bundesweice (ohne West-Bcrlin und Saarland) Erfassung der rechiskräflugen Ab-und Verurteilungen von NS-Verbrechen nach KRG 10 liegt nur fur die Jahre 1950/g) vor; vgl. Seaustik der Bundesrepublik Deutschland, Bd.110: Die Knminalieà in den Jahicen 1910 und igs 1. Daraus gehi hervor, daß in diesen beiden Jalaren zusammen noch 1866 Personen nach KRG 10 angeklage, aber nur 620 , d.h. jeder Dritc venureilt wurde. Diese Statıstlk um die Jahre 1946-1949 zu engänzen ist unmóglich, da die Statisuschen Landesamer über keın (fur Bayern, Bremen, Wurtemberg-Hohenzollern, Saarland) oder nur ìber sehr unvollständiges (für Wüntemberg-Baden: Hessen, Sud-Baden, Hamburg, Schleswsg-Holstein) Zahlenmarerial zum KRG 10 verfügen.

Für Bcrlin (ab 1950 West-Berlin) sind folgendc Verurcilungszahlen cmureclbar: 1946: 3: 1947: 71 : 1948: keine Angabe; 1949: 97; 1950: 79; 1951: 31. Quellen: Wegen Verbruchen oder Vergehen rechiskrälrig abgeurecilue Personen 1946, 1947, 1949. Wegen Verbrechen oder Vergchen rechiskràfug abgeureilte Personen nach der Aft der Entscheidung in West-Berlin r950; Berliner Statustik, Sonderheft 2\}, Die rechtskrältıg abgeurecileen Personen in West-Berlin 1950, Juni 1952; Wegen Verbrechen ader Vergehen recheskrä́fıg abgeuncilee Personen nach der An der Delikte igst. 
Wie die Verurceilung von NS-Verbrechen insgesamt erfolgce auch die Verurreilung nach KRG to in $z$ wei Phasen. In der ersten Phase von $1947-1949$ ist ein steciges Ansteigen, ab 1949 ein Zurückgehen der Verurteilungen nach KRG 10 zu verzeichnen (Abb. 1). Von 1947-1951 sind in den Ländern Berlin, Nordrhein-Westfalen, Niedersachsen und Rheinland-P\{alz 1980 Personen verurteilt worden. 1369, das sind $69,2 \%$, wurden in den ersten Jahren von 1947-1949 verurteilc.

Abb. I: Jahresgliederung der rechıskrältıgen Verurtcilungen von NS-Verbrechen durch Gerichee insgesame und nach KRG 10

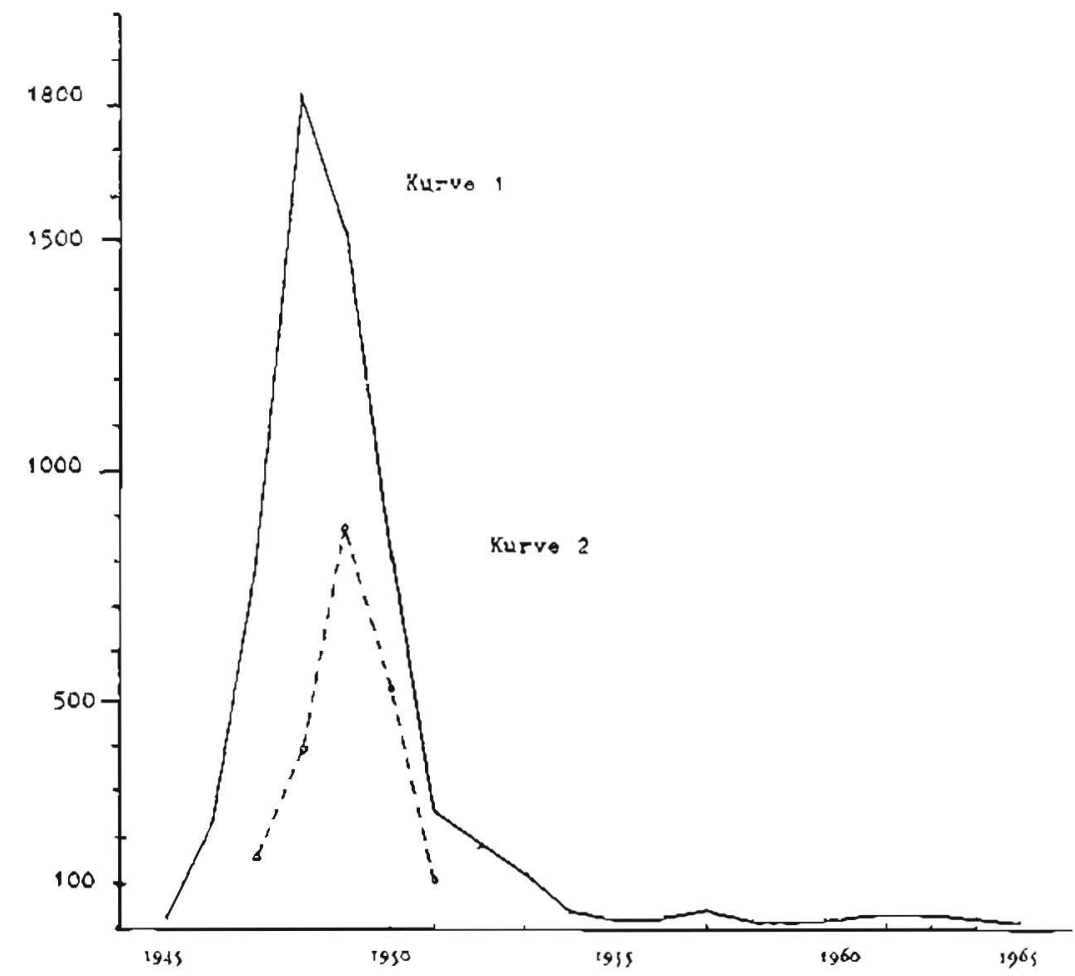

Kurve : Jahresgliederung der rechtskräfrigen Verurteilungen durch Gerichte in der Bundesrepublik

Kurve 2: Jahresgliederung der rechtskräfig nach KRG 10 Verurteilien durch Gerichse der Länder Nord̈rhcin-Westf., Berlin, Nicdersachsen und Rhejnland-Pfalz

Das Ansteigen der Verureilungszahlen von 1947-1949 ist weniger auf die wachsende Bereitschafi zur Ahndung von NS-Verbrechen zurückzuführen als aul die zunelımende Funkrionsłähigkeit der Justiz, die ab $194 \$$ die unerlcdigren Verfahren van 1945-1947 aufarbcitete. Mehr Aufschluß über die Bereitschaft zur Ahndung van NS-Verbrechen gibt dic Verurteilungsquore, $d$. h. das Verhältnis der eingcleiteten Verfahren zu den Verureilungen (vgl. Tab. $1-3$ ). Diese is in Rheinland-Pfaliz $1947 / 48$ mit $69 \%$ bow. $60 \%$, in Niedersachsen 1947 und 1949 mit 100 bzw. $50 \%$ und Nordrhein-Westfalen $1948 / 49$ mit ss bzw. $62 \%$ am höchsten. In der zweiten Phase ab Anfang 1950 bis Ende 1951 sinkt die Verurteilungsquote auf durchschnittlich 30\%. Gleichzeitig steigt die Verfahrenseinstellungsquote 1950/51 auf durchschnictich $34 \%$ in Rheinland-Pfaly, auf $44 \%$ in Niedersachsen und auf $46 \%$ in Nordrhein-Westfalen. ${ }^{167}$

167 Die meiseen Verfahrenseinxtellungen wurden aut das. Gesciz über die Gelvährung von Struffreshelta

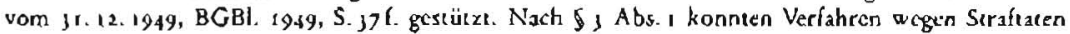


Tab. I: Die rechtskräftigen Aburteilungen nach K.RG ro durch deutsche Gerichte in Niedersachsen

\begin{tabular}{lllll|lll}
\hline Jahr & $\begin{array}{l}\text { Abgeur- } \\
\text { reilte }\end{array}$ & $\begin{array}{l}\text { Ver- } \\
\text { urteilte }\end{array}$ & $\begin{array}{l}\text { Frei- } \\
\text { spruch }\end{array}$ & $\begin{array}{l}\text { Vertahrens- } \\
\text { einsteilung }\end{array}$ & $\begin{array}{l}\text { Verurtel- } \\
\text { lungs- } \\
\text { quote } \%\end{array}$ & $\begin{array}{l}\text { Frcispr- } \\
\text { quote } \\
\%\end{array}$ & $\begin{array}{l}\text { Einstel- } \\
\text { lungsquote } \\
\%\end{array}$ \\
\hline 1947 & 15 & 15 & keine Angabe & 100 & 0 & 0 \\
1948 & 34 & 11 & 22 & 1 & 32 & 65 & 3 \\
1949 & 203 & 102 & 94 & 7 & 50 & 46 & 4 \\
1950 & 244 & 105 & 56 & 83 & 43 & 23 & 34 \\
1951 & 129 & 34 & 27 & 68 & 26 & 21 & 53 \\
\hline
\end{tabular}

Quelle: Dic Knmınaluat to Niedersiclisen 194\$-50. 1950 1951

Tab. z: Dic recheskräfng Abgeurteilten rach KRG io durch deutsche Gericlute in Nord́rbeinWestialen

\begin{tabular}{|c|c|c|c|c|c|c|c|c|}
\hline Jahre & $\begin{array}{l}\text { Abgeur- } \\
\text { tciltc }\end{array}$ & $\begin{array}{l}\text { Verur- } \\
\text { reilte }\end{array}$ & $\begin{array}{l}\text { Frel- } \\
\text { spruch }\end{array}$ & $\begin{array}{l}\text { Verf } \\
\text { cins }\end{array}$ & $\begin{array}{l}s- \\
\text { davon } \\
\text { nach } \\
\text { Amnestic- } \\
\text { Gcsetic } \\
(1.12 .49)\end{array}$ & $\begin{array}{l}\text { Verurtei- } \\
\text { lungs- } \\
\text { quore } \\
\%\end{array}$ & $\begin{array}{l}\text { Freispr.- } \\
\text { quote } \\
\%\end{array}$ & $\begin{array}{l}\text { Einstel- } \\
\text { lungs- } \\
\text { quote } \\
\%\end{array}$ \\
\hline 1947 & \multicolumn{5}{|c|}{ keine Angaben } & & & \\
\hline 1948 & 350 & 192 & 194 & 4 & & ss & as & 0 \\
\hline 1949 & 584 & 364 & 210 & 10 & & 62 & 36 & 2 \\
\hline 1950 & 377 & 127 & 105 & 145 & 97 & 34 & 28 & 39 \\
\hline 1951 & 62 & 20 & 10 & 32 & 17 & 32 & 16 & $S^{2}$ \\
\hline 1952 & 7 & 0 & 1 & 6 & s & 0 & 14 & 86 \\
\hline
\end{tabular}

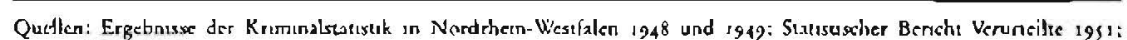
Knmunalsatistik 1952 und 1953-

Táb. 3: Die rechtskräfrig Abgeurteilten nach KRG 10 durch deutsche Gericbee in RheinlandPfalz

\begin{tabular}{|c|c|c|c|c|c|c|c|c|}
\hline Jahr & $\begin{array}{l}\text { Abgeur- } \\
\text { teiltc }\end{array}$ & $\begin{array}{l}\text { Ver- } \\
\text { urteilte }\end{array}$ & $\begin{array}{l}\text { Frei- } \\
\text { spruch }\end{array}$ & $\begin{array}{l}\text { Verfabrens } \\
\text { einstellung }\end{array}$ & $\begin{array}{l}\text { davon } \\
\text { nach } \\
\text { Amnestie- } \\
\text { Geserz } \\
(31 .\{2.49)\end{array}$ & $\begin{array}{l}\text { Verunei- } \\
\text { lungs- } \\
\text { quote } \\
\%\end{array}$ & $\begin{array}{l}\text { Freispr.- } \\
\text { quoce } \\
\%\end{array}$ & $\begin{array}{l}\text { Einstel- } \\
\text { lungs- } \\
\text { quote } \\
\%\end{array}$ \\
\hline 1947 & 16 & II & $s$ & & & 69 & 31 & \\
\hline $194^{8}$ & 141 & 85 & sI & s & & 60 & 36 & 4 \\
\hline 1949 & 369 & 174 & 194 & I & & 47 & 93 & 0,3 \\
\hline 1950 & 624 & 181 & 217 & 226 & 182 & 29 & is & 36 \\
\hline 1951 & 163 & 34 & 77 & 52 & $4^{2}$ & 21 & 47 & 32 \\
\hline
\end{tabular}

Quelten: Ergebnusse der Krmunalstaustk in Rhennland-P\{nlz 1947-1949 (Hele 7): Ergebrisse der Kriminalseatustix in Rhrunland-Píalz coso-1952 (Hofe 16)

Es erstaunt, daß sich die Abwendung der Rechisprechung vom KRG 10 erst 1950 und nicht zcitlich parallcl mit der Änderung der "Großwetrerlagc« vollzog. Der Umschwung von Neubeginn zu Kontinuität erfolgıe im wesentlichen schon 1947/

oder Ordnungswidngketten eingestelle werden, voweet diese vor dem is.9. 1949, begangen wurden und wenn nur c'une gernge Frciheiesstrale (bis zu sechs Monaten) oder einc Geldstmfe zu erivanen war. 
48. ${ }^{168}$ Aber gerade von Antang $194^{8}$ bis 1949 verurceilte die deutsche Justiz vermehrt nach KRG ro. Diese Restauration mic Zeitverschiebung könnte mit der bindenden Kraft der Rechtsprechung des $\mathrm{OGH}$ zu erklären sein, der die Untergerichte wiederholt zur Anwendung des KRG 10 ermahnte.

Nachdem 1950/51 fast die Hälfte der Verfahren eingestellt wurden, der OGH zu Ende seiner Täigkeit die Anwendung des KRG ro vermicd ${ }^{169}$ (ab 30. 5. 1950 nur noch 6 Urteile zum KRG 10) und der $B G H^{1}$, aus "Gründen, die sich einer öffentlichen Erörtcrung entziehen ${ }^{\prime 1}$ "' über eingelegte Revisionen zum KRG 10 nicht entschied, war hinsichtlich der Verfolgung von Verbrechen gegen die Menschlichkeit ein Zustand eingetreten, der nobjektiv an der Grenze einer Justizverweigerung ${ }^{\alpha}$ lag. ${ }^{172.173}$

$168 \mathrm{Vgi}$. Fn. 10.

169 Stork, Dic Rechesprechung des Obersten Genchtshofs fur dic bnusche Zone in Siralsachen, Tübingen 1969, S. 1 1: Schuliz, Rundschau. Blick in dic Zet2, MDR 1951, S. 406.

1 \% Er lösce in Oktober 1950 den OGH ab.

17 r Hulle. Aus der Rechisprechung dox BGH in Sirsfsachin, NJW r951, S. 295 (296).

172 Schuliz, $(\Gamma \cdot n .169)$, S. 406.

17) Dic Jusuzverweigerung war unsofem von Erfolg gekrönt, als dic brutusche Militärregsenung mul der Verordnung Nr. 234 und die franzósısche Militürregierung mil der Verordnung Nr. 171 im 31.8 .1951 die Ermächugung deutscher Gerchte zur Abureilung von NS-Verbrechicn nach KRG ro zurucknahmen, Amusbl. d. Alliierien Hohen Kommission 1951, S. 11371.

Das KRG 10 selbst hat erst durch \& 2 des $x$ Ersren Gesetzes zur Aufhebung des Beszizungsreclizs." vom 30. 5. 1964 seune Wirksamkcil un Geltungsberesch des Grundgescizes verloren, BGBI. 1, S. 437. Sele dur Rücknahme der Ermischigung am 31.8.1951 wurden NS-Verbrechen ausschlicBlich nach dculschem Rechl abgeureilt.

Gleıchzesig wurde versuch, die Ahndung von NS-Verbrechen überhaupt zu beenden, unders bchaup. (ci wurdc, daß durcb dic Rücknahme der Ermächeıgung ein ProzcBhindernus cingutreten se1, das dic Einstellung dar NS-Verfahren nacls 2062 Abs, a StPO emogliche, OLG Hamburg 8. 12. 1991, MDR 1951, S. 183: LG Lübeck 24.11. 1951, un: Ruucer, (F-n, 110), Bd. IX, S. 169; Schultz, (Fn. (69), S. 410. Hier var der politusche Wunsch, NS-Verbrechen überhaupe niche mehr zu ahnden, offensichelich Herr der jurisuschen Argumentation. Ein Verlahrenshinderms ist an Umsuand, der den Erlaß eines Sachureils ausschlicßc. Ein solcher Umsand is durch die Verordnung 234 bzw. i 71 niche eingctecten, denn dic Bestralung der Tae war in den merseen Füllen weiterhın nach deutschem Serairechı möglich, so d.A eune Einstellung nach $\$ 206 a$ StPO micht in Frage kam. 